\title{
Th17 cells are refractory to senescence and retain robust antitumor activity after long-term ex vivo expansion
}

\author{
Jacob S. Bowers, ${ }^{1,2,3}$ Michelle H. Nelson, ${ }^{1,2,3}$ Kinga Majchrzak, ${ }^{1,2,3,4}$ Stefanie R. Bailey, ${ }^{1,2,3}$ \\ Baerbel Rohrer, ${ }^{5,6}$ Andrew D.M. Kaiser, ${ }^{7}$ Carl Atkinson, ${ }^{1}$ Luca Cattinoni, ${ }^{8}$ and Chrystal M. Paulos ${ }^{1,2,3}$ \\ ${ }^{1}$ Department of Microbiology and Immunology, ${ }^{2}$ Department of Dermatology, ${ }^{3}$ Department of Surgery, Medical University \\ of South Carolina, Charleston, South Carolina, USA. ${ }^{4}$ Department of Physiological Sciences, Faculty of Veterinary \\ Medicine, Warsaw University of Life Sciences, Warsaw, Poland. ${ }^{5}$ Department of Ophthalmology, Medical University of \\ South Carolina, Charleston, South Carolina, USA. ${ }^{6}$ Ralph H. Johnson VA Medical Center, Charleston, South Carolina, USA. \\ ${ }^{7}$ Center for Cancer Research, ${ }^{8}$ Experimental Transplantation and Immunology Branch, National Cancer Institute (NCI), NIH, \\ Bethesda, Maryland, USA.
}

Adoptive immunotherapy for solid tumors relies on infusing large numbers of T cells to mediate successful antitumor responses in patients. While long-term rapid-expansion protocols (REPs) produce sufficient numbers of $\mathrm{CD8}^{+} \mathrm{T}$ cells for treatment, they also cause decline in the cell's therapeutic fitness. In contrast, we discovered that IL-17-producing CD4+ $T$ cells (Th17 cells) do not require REPs to expand 5,000-fold over 3 weeks. Also, unlike Th1 cells, Th17 cells do not exhibit hallmarks of senescence or apoptosis, retaining robust antitumor efficacy in vivo. Three-weekexpanded Th17 cells eliminated melanoma as effectively as Th17 cells expanded for 1 week when infused in equal numbers into mice. However, treating mice with large recalcitrant tumors required the infusion of all cells generated after 2 or 3 weeks of expansion, while the cell yield obtained after 1-week expansion was insufficient. Long-term-expanded Th17 cells also protected mice from tumor rechallenge including lung metastasis. Importantly, 2-week-expanded human chimeric antigen receptor-positive (CAR+) Th17 cells also retained their ability to regress human mesothelioma, while CAR+ Th1 cells did not. Our results indicate that tumor-reactive Th17 cells are an effective cell therapy for cancer, remaining uncompromised when expanded for a long duration owing to their resistance to senescence.

Conflict of interest: The authors have declared that no conflict of interest exists.

Submitted: September 19, 2016 Accepted: January 19, 2017 Published: March 9, 2017

Reference information: JCI Insight. 2017;2(5):e90772. https:// doi.org/10.1172/jici.insight.90772

\section{Introduction}

Adoptive cell therapy (ACT) is a powerful means for augmenting a patient's immunity to cancer via the ex vivo selection, expansion, and enhancement of autologous tumor-reactive $\mathrm{T}$ cells. An advantage of ACT is the potential for complete and durable responses executed by memory T cell populations (1). In patients with metastatic melanoma, this therapy achieves remarkable outcomes for patients, with objective responses of $54 \%$ and complete remissions of $24 \%(2,3)$.

To accomplish durable results in patients, ACT requires the infusion of large numbers of tumor-reactive $\mathrm{T}$ cells $\left(\sim 10^{10}\right.$ cells) (4-10). Most protocols for generating vast $\mathrm{T}$ cell numbers require their expansion for up to 3 months with at least 1 CD3-dependent reactivation (8-10). However, ex vivo expansion works in direct opposition to maintaining antitumor efficacy because the cytotoxic $\mathrm{CD} 8^{+} \mathrm{T}$ cells used in clinical trials lose their potency when extensively expanded ex vivo. This loss of antitumor efficacy is due to a reduced capacity to persist in vivo once $\mathrm{CD} 8^{+} \mathrm{T}$ cells reach terminal differentiation (11). Additionally, this effect occurs rapidly, as even highly potent IL-12-primed CD8 ${ }^{+} \mathrm{T}$ cells lose antitumor efficacy in as little as 1 week of expansion (12). Rapid-expansion protocols (REPs) also compromise the integrity of human tumor-infiltrating lymphocytes (TILs), while TILs that undergo shorter expansion ex vivo persist longer $(1,13-15)$.

Although investigators have developed strategies to prevent $\mathrm{T}$ cell differentiation ex vivo $(16,17)$, another potential avenue is to circumvent this dilemma using a $\mathrm{T}$ cell subset relatively refractory to senescence. We used $\mathrm{CD}^{+} \mathrm{T}$ cells with a TCR specific for tyrosinase-related protein-1 (TRP-1, a shared melanocyte/ melanoma antigen) that were polarized to a Th17 phenotype ( $\mathrm{CD}^{+} \mathrm{T}$ cells that express the transcription 
factor ROR $\gamma$ t and secrete IL-17). In murine models of melanoma, TRP-1 Th17 cells offer an alternative to melanoma-reactive pmel-1 CD8 ${ }^{+} \mathrm{T}$ cells, as they mediate a potent response against aggressive melanoma in mice even without antigen vaccination and in vivo IL-2 support required in pmel-1 CD8 ${ }^{+} \mathrm{ACT}$ models (18-20). This remarkable Th17 antitumor response occurs in part by enhancing $\mathrm{CD}^{+} \mathrm{T}$ cell responses against transformed cells (21-24) and via their ability to directly lyse tumors $(25,26)$. In both cases, Th17 cells exert superior antitumor immunity compared with Th1 and other CD4 ${ }^{+} \mathrm{T}$ cell subsets (18), as well as superior enhancement of CD8 ${ }^{+} \mathrm{T}$ cells (23). Also, since Th17 cells show durable efficacy in vivo and have stem memory properties $(27,28)$, we posited that unlike $\mathrm{CD}^{+} \mathrm{T}$ cells, Th17 cells would retain their antitumor capacity even after long-term ex vivo expansion to clinically relevant doses.

We found that, even without a REP, Th17 cells were capable of robust growth for 21 days ex vivo, yielding $\sim 5,000$ times the initial $\mathrm{CD}^{+} \mathrm{T}$ cell numbers. Additionally, while exhibiting a $\mathrm{CD} 44^{\text {hi }} \mathrm{CD} 62 \mathrm{~L}^{\text {lo }}$ effector memory phenotype, Th17 cells gained nuclear Tcf7 throughout in vitro culture, indicating active and preserved stem memory signaling. Unlike Th1 or $\mathrm{CD}^{+} \mathrm{T}$ cells, Th17 cells retained their ability to eliminate melanoma tumors over 2 weeks of expansion. Th17 cells were resistant to senescence indicated by retention or upregulation of CD127, CD28, and CD27 along with nominal expression of KLRG1 and TIM3. In contrast, Th1 cells expressed exhaustion markers and higher levels of checkpoint receptors and were far more apoptotic than Th17 cultures at any time point of expansion. Importantly, Th17 cells expanded for either a 7- or 21-day duration ex vivo were equally able to persist in the tumor-bearing host after transfer. Despite similar antitumor potency between 7- and 21-day Th17 cells on a per-cell basis, we hypothesized that mediating curative responses in mice with large, nearly terminal tumors would require the full yield of Th17 cells generated from 3 weeks of culture. We found that treatment with 2- or 3-weekexpanded Th17 cells rapidly and completely eradicated aggressively growing tumors, while the full yield of 1-week-expanded Th17 cells was insufficient to control these tumors. Of clinical significance, human Th17polarized $\mathrm{CD} 4^{+}$cells also exhibit marked durability in antitumor potency compared with Th1-polarized cells when expanded for either 7 or 12 days. Our analysis of all parameters of Th17 cell ex vivo preparation for ACT (in vitro time of expansion, cell yield, T cell subset, and size of tumor) indicates that tumor-reactive Th17 cells represent a cell product that can be reliably applied to treat highly aggressive solid tumors.

\section{Results}

ACT with Th17 cells still requires transferring large numbers of T cells to clear tumors. The effectiveness of ACT is thought to depend on the transfer of large numbers of tumor-specific T cells (11). However, because Th17 cells mediate potent antitumor responses in mice and have durable stem memory properties, we hypothesized that we could titrate down the number of Th17 cells infused into mice without impairing tumor clearance. To test this idea, TRP-1 CD4+ T cells were polarized to a Th17 phenotype and expanded ex vivo for 1 week. This protocol generated cells with expression of the Th17 transcription factor ROR $\gamma$ t, high IL-17 production, and nominal Tbet (the Th1 transcription factor) and IFN- $\gamma$ (Figure 1, A and B). We infused decreasing doses of Th17 cells $\left(4.5,1.5\right.$, or $0.5 \times 10^{6}$ cells/mouse) into C57BL/6J mice with established B16F10 melanoma preconditioned with nonmyelablative (5 Gy) total body irradiation (Figure 1C). While the highest number of Th17 cells $\left(4.5 \times 10^{6}\right)$ eliminated tumors in most mice, tumor responses and overall survival declined in mice treated with fewer Th17 cells (Figure 1, C and D). Thus, despite their enhanced antitumor efficacy and a stem memory profile, Th17 cells require a sufficient number of transferred cells for successful ACT.

REP decreases the antitumor capacity and recall responses of Th17 cells. Since large numbers of Th17 cells are required to eradicate tumors, we tested whether extending the in vitro culture time for another week using a REP with TCR reactivation would improve their yield. To test this, as presented in Figure 2A, Th17 cells were expanded for 2 weeks, with the REP culture undergoing TCR reactivation using TRP-peptide-pulsed feeder cells at 1 week. Interestingly, Th17 cells only activated once (primary expansion) could effectively expand for 2 weeks to similar yields as REP Th17 cells (Figure 2B). By day 14, both Th17 and REP Th17 cells were mainly $\mathrm{CD} 44^{\mathrm{hi}} \mathrm{CD} 62 \mathrm{~L}^{\text {lo }}$ effector memory (Figure 2C). However, REP Th17 cells were slightly compromised in their ability to regress tumors and promote survival in mice (Figure 2, D and E). This was not due to increased levels of apoptosis, KLRG1, PD1, or TIM3 on the REP Th17 cells compared with Th17 cells (Supplemental Figure 1, A-C; supplemental material available online with this article; https:// doi.org/10.1172/jci.insight.90772DS1). However, while the frequency of donor Th17 cells in the spleen and tumors of surviving mice was similar to the frequency of REP Th17 cells 40 days after treatment (Figure 2, F 

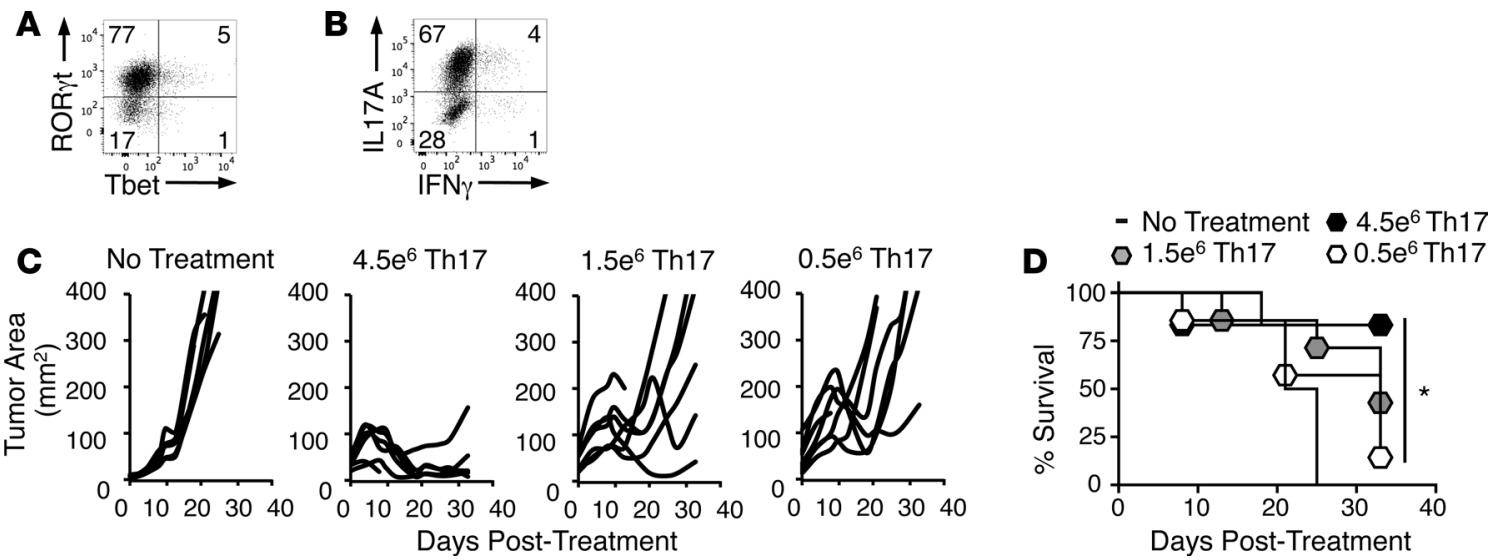

Figure 1. High numbers of transferred Th17 cells are required for successful antitumor response. (A) ROR $\gamma \mathrm{t}$ and Tbet expression by Th17 cells after 7 days of culture; representative of 3 independent experiments. (B) IL-17A and IFN- $\gamma$ expression by Th17 cells after 7 days of culture; representative of 5 independent experiments. (C) Th17 cells cultured ex vivo for 9 days were administered to mice with B16F10 melanoma after nonmyeloablative (5 Gy) total body irradiation 1 day prior to treatment with the indicated Th17 cell doses; $n=6-7$ mice/group, representative of 3 independent experiments. (D) Percentage survival of mice treated with $4.5,1.5$, or $0.5 \times 10^{6}$ Th17 cells/mouse. Kaplan-Meier curves compared by log-rank test. ${ }^{*} P<0.05$.

and G), memory recall against TRP-1 antigen by mice treated with REP donor Th17 cells was significantly impaired compared with mice treated with Th17 cells in an in vivo cytotoxicity assay (Figure $2 \mathrm{H}$ ). Thus, REPs appear to compromise the antitumor efficacy and antigen-recall responses of donor Th17 cells.

Th17 cells become less activated but not terminally differentiated during ex vivo expansion. We next sought to determine how long Th17 cells could be expanded in vitro without losing antitumor efficacy. We found that Th17 cells could expand for 3 weeks without TCR reactivation (Figure 3A). While tyrosinase-specific $\left(\mathrm{V} \beta 14^{+}\right) \mathrm{CD} 4^{+} \mathrm{T}$ cells have a naive phenotype $\left(\mathrm{CD} 44^{\mathrm{lo}} \mathrm{CD} 62 \mathrm{~L}^{\text {hi }}\right)$ when isolated from the spleens of TRP-1 transgenic mice, Th17 cells exhibited an effector memory phenotype with high CD44 and low $\mathrm{CD} 62 \mathrm{~L}$ expression as early as 1 week after activation, which prevailed throughout culture (Figure 3B). This phenotype corroborates previous studies with human and murine Th17 cells $(18,27)$. However, Th17 cells expressed Tcf7 (a downstream product of active $\beta$-catenin signaling), throughout culture (Figure 3, C and D; see complete unedited blots in the supplemental material), indicating sustained capacity for self-renewal and persistence (29).

Because we were not using a REP to expand the Th17 cells, we expected that the cells would gradually become quiescent and downregulate activation markers as they were expanded for 21 days in vitro. As expected, activation markers CD69 and CD25 (IL-2R $\alpha$ ) were initially high 1 week after stimulation but progressively declined (Figure 3E). Similarly, Th17 cells initially expressed high levels of ICOS, which is an important costimulatory molecule that supports their function and maintenance $(19,30)$, while the costimulatory receptor 4-1BB was only increased moderately above preactivation levels at the early Th17 time point. In contrast, CD127 (IL-7R $\alpha$ ) was maintained on Th17 cells expanded for 3 weeks, suggesting a persistent responsiveness to IL-7 (31). The costimulatory ligand CD28 was similarly retained. CD27, which is associated with adaptive memory responses (32), increased between week 1 and week 3 (Figure 3E). Importantly, the retention of $\mathrm{CD} 127$ and $\mathrm{CD} 28$, and the increased expression of CD27 during expansion, may indicate that Th17 cells are resistant to the senescence that naturally occurs in other T cell subsets (33).

Long-term-expanded Th17 cells retain antitumor potency and persist after transfer. Based on evidence that Th17 cells maintained active Wnt/ $\beta$-catenin signaling (Figure 3, C and D), and the pattern of chemokine and costimulatory receptor expression we observed throughout culture, we posited that Th17 cells (in contrast to $\mathrm{CD}^{+} \mathrm{T}$ cells; see ref. 11) would retain antitumor activity as they expanded long term in vitro. To compare Th17 cell antitumor efficacy over ex vivo culture time on a per-cell basis, Th17 cells were expanded for 3 weeks, with a portion of cells cryopreserved at either (a) early (day 6 or 7), (b) intermediate (days 12-14), or (c) late (days 18-21) time points in culture. Th17 cells were then transferred at equal numbers $\left(2 \times 10^{6}\right.$ Th17 cells/mouse) from each time point into lymphodepleted mice bearing established melanoma. Note that for late Th17 culture, the infusion product represented a mere 1/2,500 of their total cell count. However, regardless of the duration of in vitro expansion, Th17 cells killed tumors (Figure 3F) and supported long-term survival (Figure $3 \mathrm{G}$ ) in the majority of mice. 
A
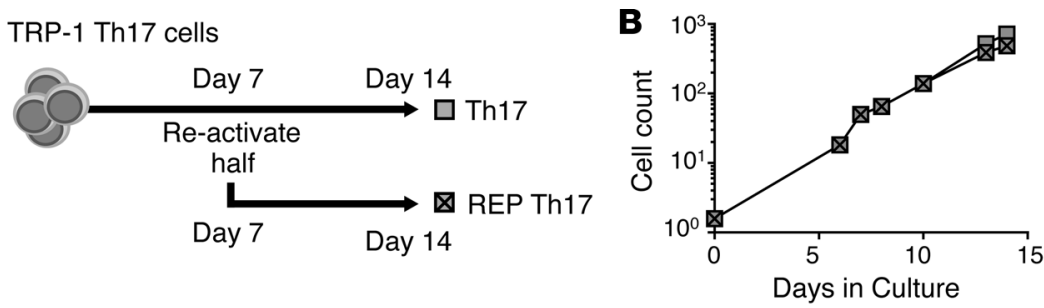

D

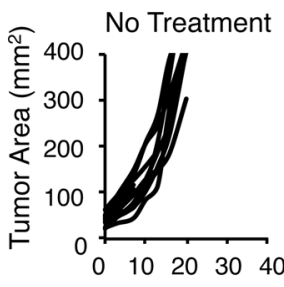

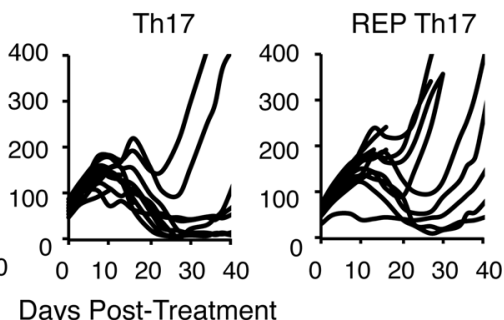

Days Post-Treatment

\section{G}

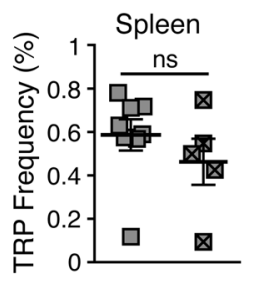

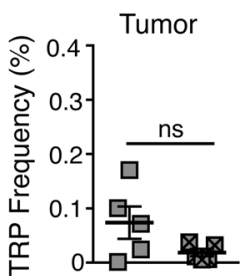

H

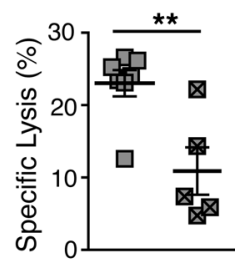

Figure 2. Rapid-expansion protocol (REP) does not improve Th17 yield and reduces antitumor efficacy. (A) Schematic for growth of Th17 and REP Th17 cultures. (B) Yield of Th17 and REP Th17 cells after 2 weeks of culture, representative of 3 independent cultures. (C) CD44 and CD62L memory marker expression on Th17 or REP Th17 cells on day $15, n=3$ independent cultures. (D) Mice with B16F10 were treated with $2 \times 10^{6}$ Th17 cells or REP Th17 cells after 5 Gy total body irradiation; $n=10$ mice/group, representative of 2 independent experiments. (E) Percentage survival of cohorts treated with Th17 or REP Th17 cells; Kaplan-Meier curves compared by log-rank test. (F) Mean frequency ( \pm SEM) of donor Th17 or REP Th17 cells in spleen of treated mice; $n$ $=5-8$ mice/group. (G) Mean frequency ( \pm SEM) of donor Th17 or REP Th17 cells in tumors of treated mice; $n=5$ mice/group. (H) Memory response of donor Th17 or REP Th17 cells evaluated by an in vivo cytotoxicity assay reported as mean percentage specific lysis ( \pm SEM); $n=5-8$ mice/group. Donor Th17 versus REP Th17 mean frequency and percentage specific lysis compared by Student's $t$ test. ${ }^{* *} P$ $<0.01$. ns, not significant. TRP, CD4 ${ }^{+} \mathrm{T}$ cells with a TCR specific for tyrosinase-related protein-1.

$\mathrm{T}$ cell persistence correlates with enhanced treatment outcome in melanoma patients given ACT (1). Thus, the similar antitumor efficacy mediated by early- or late-culture Th17 cells in mice suggested that these cells - regardless of time in vitro - would engraft and persist in mice at similar levels. To test this idea, we followed donor TRP-1 CD45.2+ Th17 cells expanded for 7 (early) or 21 days (late) in CD45.1 $1^{+}$ recipient mice over 28 days. Early and late Th17 cells were present at equivalent numbers after treatment at 7,14 , and 28 days in the spleen and after 7 days in the tumor (Figure $3 \mathrm{H}$ ). The eye is a common off-site target for $\mathrm{T}$ cell models specific for melanocyte-associated antigens (i.e., tyrosinase) because of melanocytes in the pigment epithelium (34). Here, too, we detected early and late Th17 cells at comparable amounts in melanoma-bearing mice (Figure $3 \mathrm{H}$ ).

In ACT clinical trials, a donor T cell with longer telomeres persists better in melanoma patients and is associated with improved survival $(13,14)$. We hypothesized that preserved telomere length during expansion both in vitro and in vivo might be a mechanism by which Th17 cells, at any stage of expansion, preserved their antitumor efficacy after in vitro expansion. Telomere length only slightly decreased in Th17 cells as they expanded in vitro (Figure 3I). However, Th17 cells from both early and late cultures maintained comparable telomere length 4 weeks after ACT (Figure 3J). Thus, along with Tcf7, another partial explanation for the durable antitumor response of Th17 cells may be their maintenance of telomere length in vitro and in vivo. Collectively, our data reveal that Th17 cells expanded for 3 weeks retain antitumor efficacy compared with 1-week-old counterparts, and that this may be due to persistent stem signaling and telomere length which sustain the in vivo persistence of these cells.

Th17 cells undergo polyfunctional conversion during expansion but do not change their interaction with host immune cells. We noticed that even without inflammatory signaling such as IL-12, which drives Th17 cell conversion to a Th1-like phenotype (termed Th17 plasticity; see refs. 35-37), Th17 cells became progressively polyfunctional. As seen by ELISA performed 18 hours after peptide-dependent reactivation, IL17A and IL-17F decreased in concert throughout culture, IL-22 was lost rapidly after 1 week, and IL-2 and IFN- $\gamma$ production increased during ex vivo expansion (Supplemental Figure 2A). This correlated with reduced levels of nuclear ROR $\gamma \mathrm{t}$ and STAT3, although both molecules persisted throughout culture (Supplemental Figure 2, B and C). Additionally, Th17 cells maintained nuclear STAT1, while Tbet was not detected (Supplemental Figure 2, B and C). We suspected that the increased polyfunctionality of 

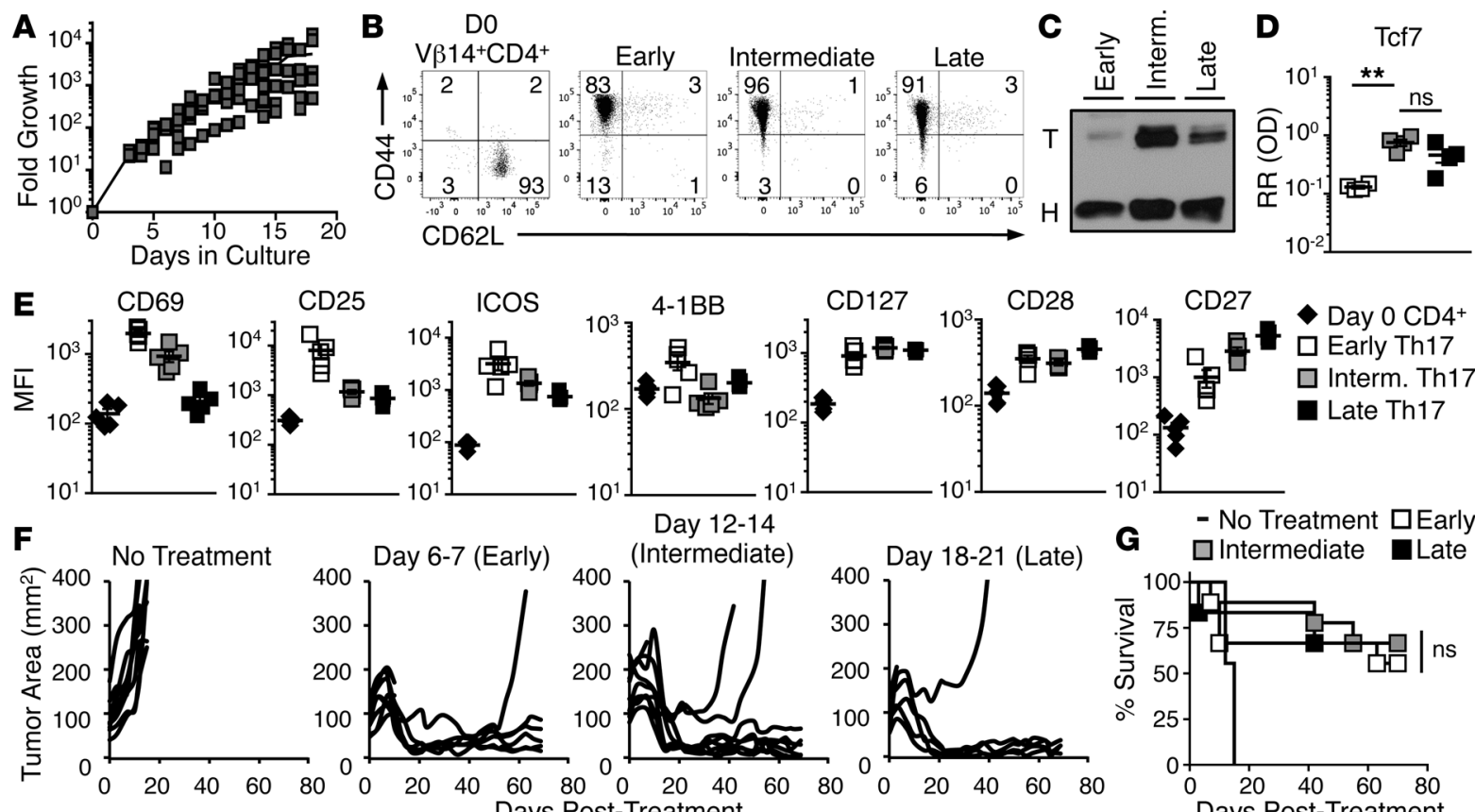

Day $12-14$
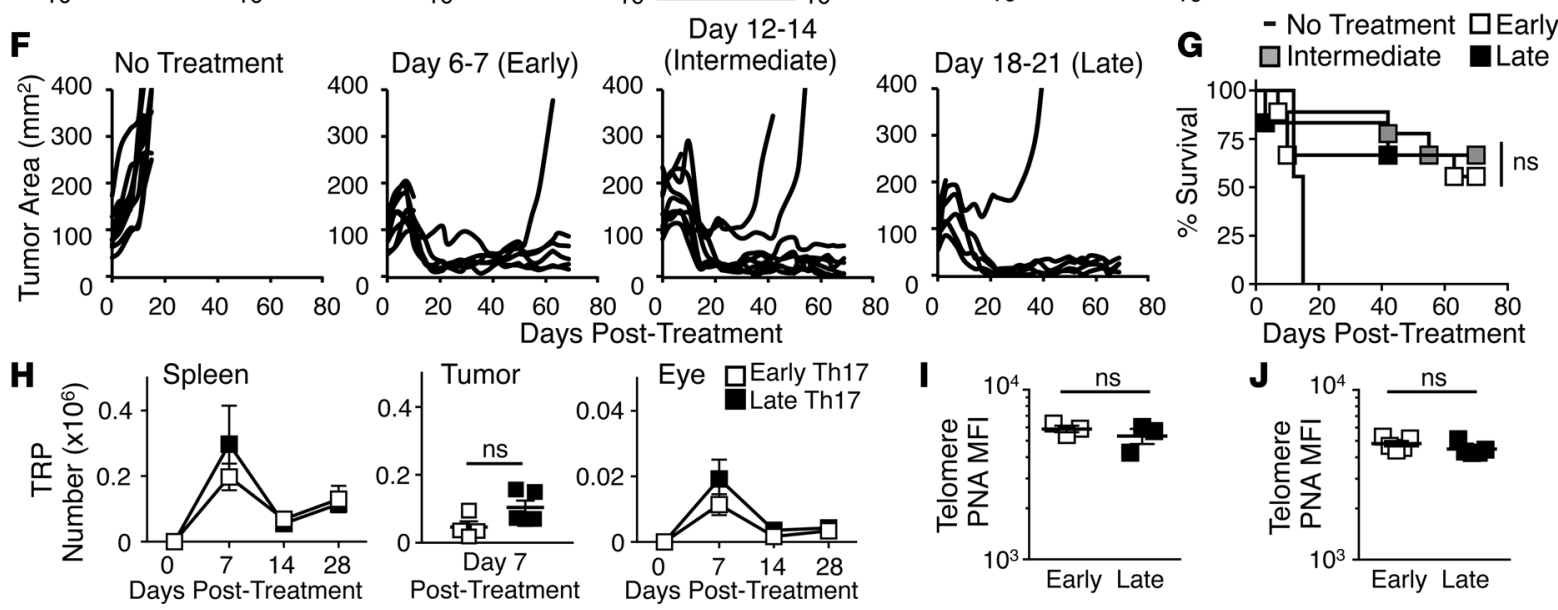

Figure 3. Th17 cells expanded for 3 weeks enter quiescence but retain antitumor potency and ability to engraft. (A) Growth of Th17 cells in culture; $n=$ 7 independent cultures. (B) CD44 and CD62L memory marker expression on V $\beta 14^{+} \mathrm{CD} 4^{+} \mathrm{T}$ cells before polarization and Th17 cells at different time points in culture analyzed via flow cytometry; representative of 3-5 cultures. (C) Western blot analysis of nuclear protein from 7-, 14-, and 21-day-expanded Th17 cells, T, Tcf7; H, histone H3; representative of 4 independent cultures. (D) Quantified Tcf7 protein relative to histone H3 (mean \pm SEM); $n=4$ independent cultures. RR (OD), relative ratio of optical density. (E) Mean fluorescence intensities (MFIs) ( \pm SEM) of cytokine and costimulatory receptors during ex vivo culture of Th17 cells assessed by flow cytometry; $n=5$ independent cultures. (F) Mice with B16F10 tumors were treated with equal numbers $\left(2 \times 10^{6}\right.$ cells/mouse) of Th17 cells from the early, intermediate, and late time points of ex vivo culture following 5 Gy total body irradiation 1 day prior; $n=8-9$ mice/group, representative of 4 independent experiments. (G) Percentage survival of mice treated in Figure 3F; $n=8-9$ mice/group, Kaplan-Meier curves compared by log-rank test. (H) Mean number ( \pm SEM) of donor Th17 cells from early or late culture time points in host C57BL/6 spleen, tumor, and eye at indicated days after treatment; $n=4-5$ mice, representative of 2 independent experiments. Mean cell numbers of early or late Th17 cells compared by Student's $t$ test. TRP, CD4 ${ }^{+}$T cells with a TCR specific for tyrosinase-related protein-1. (I) Hybridized telomere peptide nucleic acid (PNA) in Th17 cells at early and late culture time points; $n=3$ independent cultures. (J) MFI ( \pm SEM) of telomere length in donor Th17 cells 4 weeks after treatment; $n=5$ mice/ group. Telomere MFIs compared by Student's $t$ test. ${ }^{* *} P=0.0021$. ns, not significant.

3-week-expanded Th17 cells compared with 1-week-old counterparts would increase the recruitment of host immune cells in the tumor-bearing mouse to inflammatory sites. In contrast, we found that mice treated with 21-day-expanded Th17 cells actually had slightly fewer (though not statistically significant) host $\mathrm{CD} 4^{+}$and $\mathrm{CD} 8^{+}$cells in the spleens and eyes of recipient $\mathrm{CD} 45.1^{+}$mice, indicated by their higher ratio of donor Th17 to host cells (Supplemental Figure 2, D and E).

These differences between early and late Th17 cells, as well as previous data showing that Th17 cells enhance tumor-specific $\mathrm{CD} 8^{+} \mathrm{T}$ cell immunity (23), led us to question if early or late Th17 cells utilize endogenous $\mathrm{CD}^{+} \mathrm{T}$ cells to a different degree in their antitumor response. We transferred early or late Th17 cells and antibody-depleted $\mathrm{CD} 8^{+} \mathrm{T}$ cells to address this question. Interestingly, all groups had similar antitumor responses and survival rates (Supplemental Figure 2, F and G), suggesting that Th17 cells are capable of tumor lysis independent of $\mathrm{CD} 8^{+} \mathrm{T}$ cells. However, one should be conservative with this conclusion, as other reports suggest depleting host $\mathrm{T}$ cells might be beneficial to ACT by removing competition for homeostatic cytokines (38), which may have confounded the results. Thus, while long-term-expanded donor Th17 cells undergo functional conversion, they recruit and interact with host immune cells in tumorbearing mice comparably to infused Th17 cells expanded for 7 days in vitro. 
A

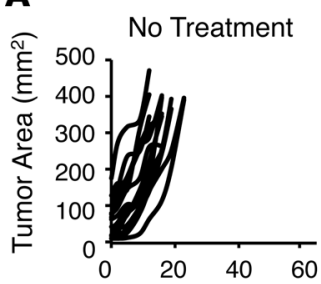

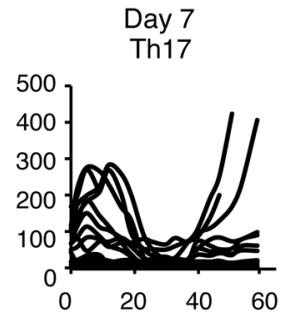

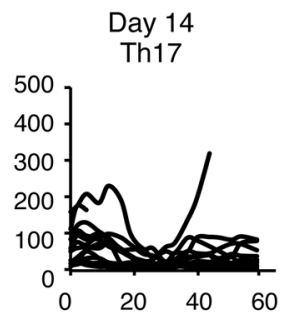

Days Post-Treatment
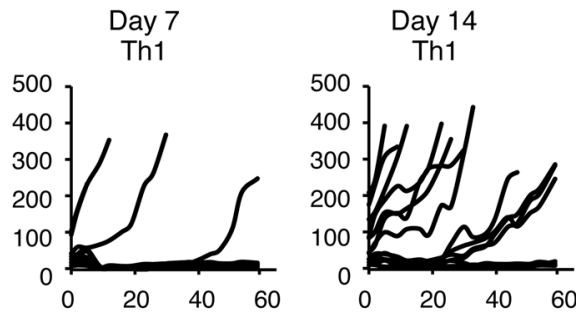

B

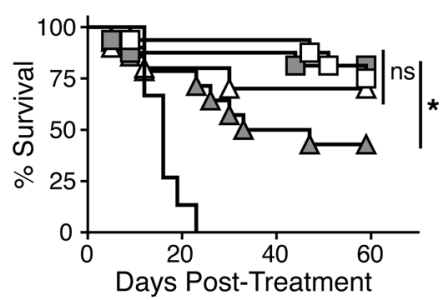

C
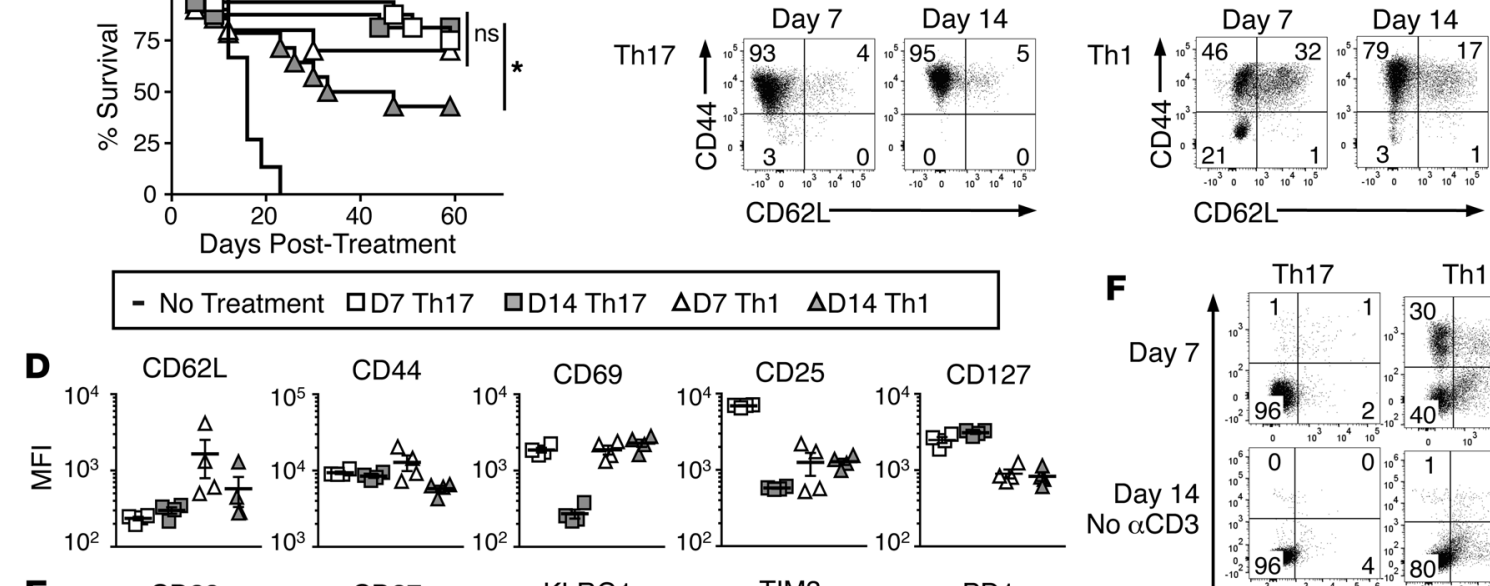

口D14 Th17 $\Delta \mathrm{D} 7 \mathrm{Th} 1 \quad \Delta \mathrm{D} 14 \mathrm{Th}$
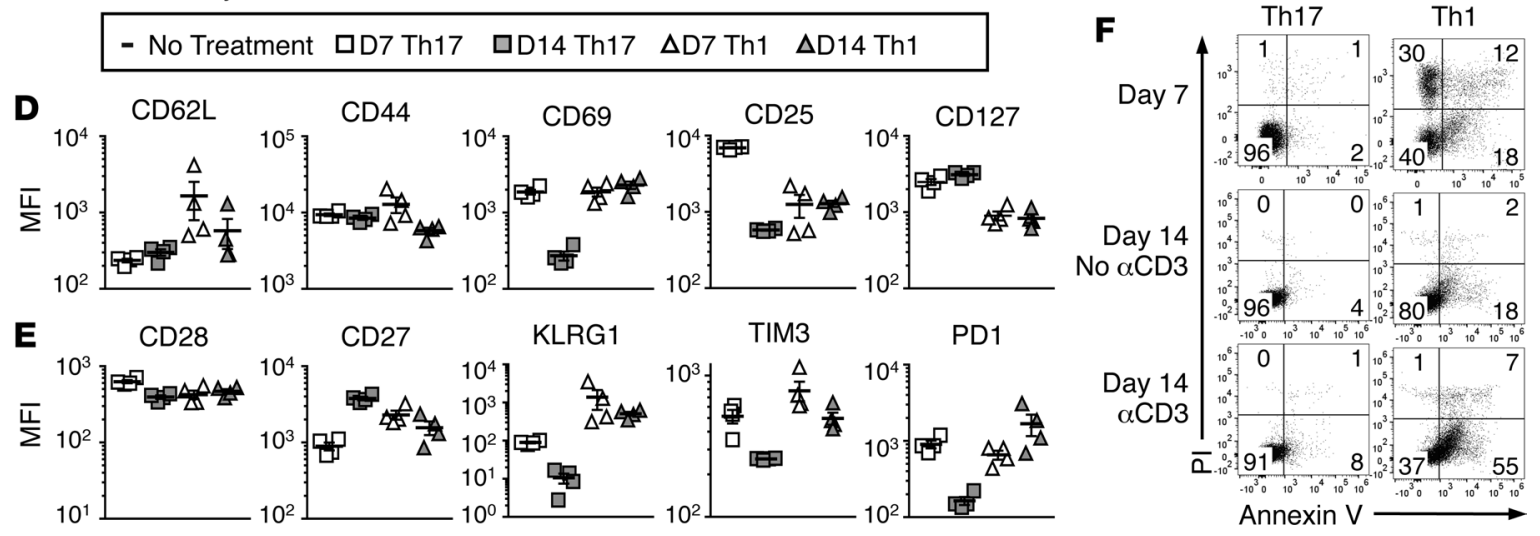

Figure 4. Th17 cells are resistant to senescence and apoptosis during ex vivo expansion compared with Th1 cells. (A) Tumor burden of mice treated with 1- or 2-week-expanded Th17 or Th1 cells compared to no treatment; $n=10-16$ mice/group, combined from 2 independent experiments. (B) Percentage survival of mice treated with 1- or 2-week-expanded Th17 or Th1 cells compared with no treatment; $n=10-16$ mice/group, combined from 2 independent experiments; Kaplan-Meier curves compared by log-rank test. ${ }^{*} P<0.05$. (C) CD44 and CD62L on Th17 and Th1 cells expanded for 1 or 2 weeks in vitro, $n=3$ independent cultures. (D-E) Mean fluorescence intensity (MFI) ( \pm SEM) of extracellular receptors during ex vivo culture of Th17 and Th1 cells assessed by flow cytometry; $n=4$ independent cultures. (F) Propidium iodide (PI) and annexin V staining on 1- or 2-week-expanded Th17 and Th1 cells. Day-14 Th17 and Th1 cells were either incubated with $1 \mu \mathrm{g} / \mathrm{ml}$ anti-CD3\& for 12 hours or left as an untreated control; $n=3$ independent cultures.

Th17 cells but not Th1 cells resist senescence and apoptosis in vitro. We next tested whether TRP-1 Th1 cells, like TRP-1 Th17 cells, could retain their antitumor efficacy after long-term ex vivo expansion. While Th17 cells cleared tumors similarly after 1 or 2 weeks of expansion, 2-week-expanded Th1 cells lost their ability to control tumor growth (Figure 4, A and B). This is similar to the loss of antitumor efficacy by CD ${ }^{+}$ T cells, which we found occurs in IL-12-primed pmel-1 CD8 ${ }^{+} \mathrm{T}$ cells even without REP. Expanding these cells for only 10 days still caused a dramatic reduction in antitumor activity compared with 4-day-expanded $\mathrm{CD}^{+} \mathrm{T}$ cells (Supplemental Figure 3, A and B). Note that the CD8 ${ }^{+} \mathrm{T}$ cells lost control of tumors even after in vitro activation (a substitute for antigen vaccination; see ref. 39) and post-transfer injections of highdose IL-2, which were not used in either our Th17 or Th1 therapies.

To understand why Th17 and not Th1 cells had durable antitumor efficacy during expansion, we surveyed expression of memory and other surface markers as well as levels of apoptosis in culture over time. Th1 cells had a significant cohort of central memory cells at 1 week of culture compared with Th17 cells, as indicated by their CD62L expression (32\% on Th1 vs. 4\% on Th17). However, by the second week, CD62L decreased on Th1 cells by half (Figure 4, C and D). Thus, CD62L expression on Th1 cells appears to correlate with their antitumor potential, similar to $\mathrm{CD}^{+} \mathrm{T}$ cells.

Unlike Th17 cells, Th1 cells maintained CD69 and CD25 expression between day 7 and day 14. Importantly, however, levels of CD25 on early Th1 cells were lower than on early Th17 cells, indicating a reduced capacity to respond to the homeostatic cytokine IL-2. Similarly, Th1 cells also had less CD127 than Th17 cells (Figure 4D). We assayed the levels of other markers that, along with homeostatic cytokine receptor levels, 
A
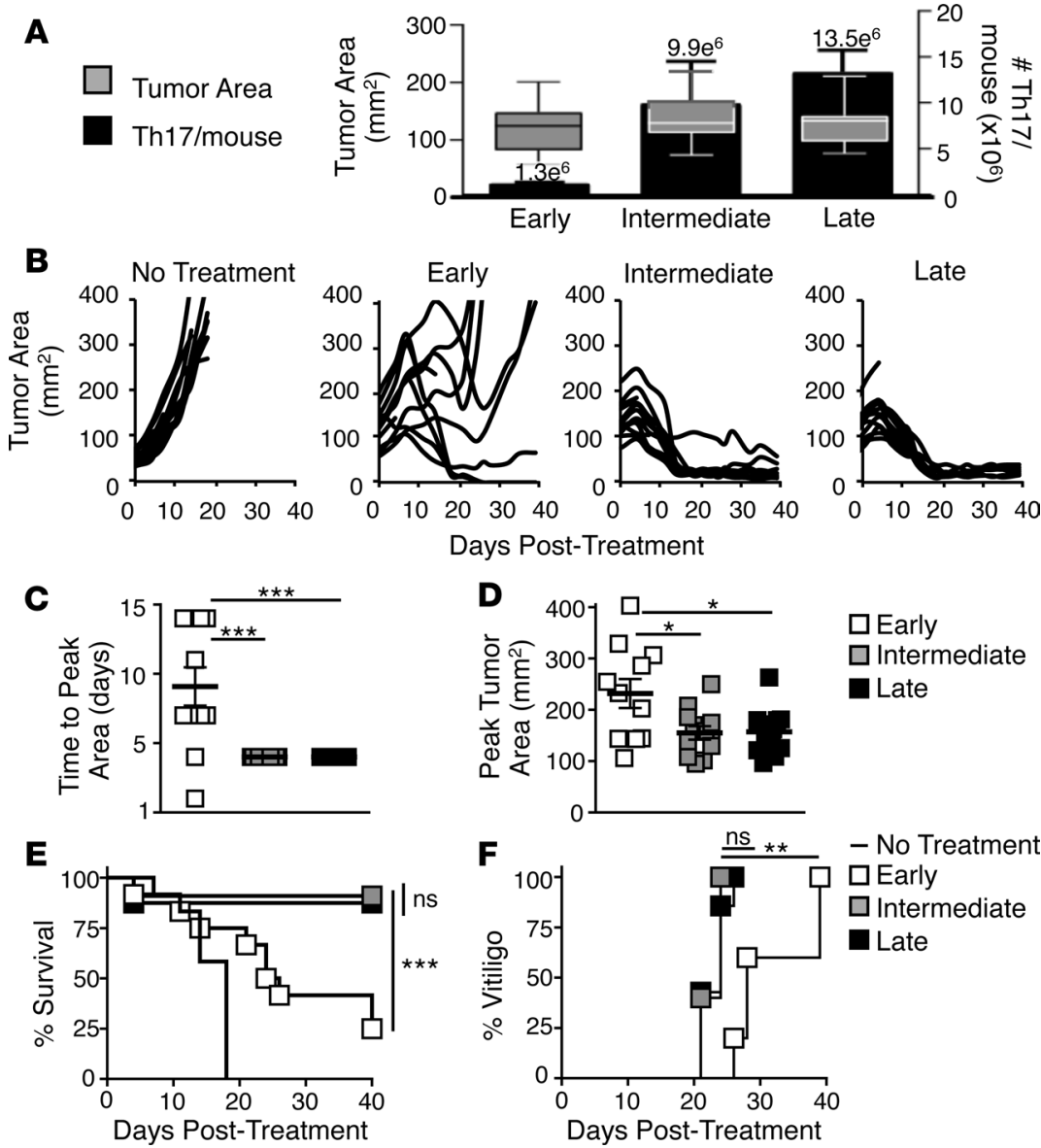

Days Post-Treatment

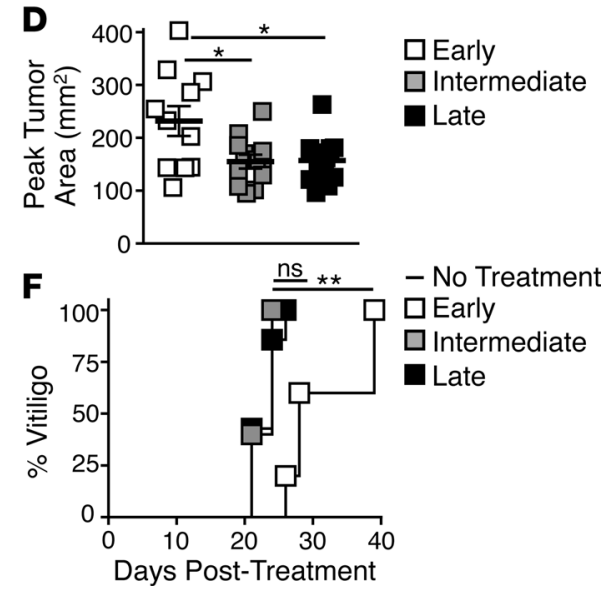

Figure 5. Successful Th17 cell adoptive cell therapy against large tumors requires the cell numbers achieved by long-term ex vivo expansion. (A) Mean ( \pm SEM) number of Th17 cells administered to mice with large (median $136 \mathrm{~mm}^{2}$ ) B16F10 tumors from parallel cultures expanded to either early, intermediate, or late culture time points and divided among 12 mice/culture. Boxes represent the 25th to 75 th quartiles and bars represent the minimum and maximum values; $n=2$ cultures. (B) Tumor control in mice treated with early-, intermediate-, or late-culture Th17 cells; $n=12$ mice/group, representative of 3 independent experiments. (C) Mean time ( \pm SEM) in days after treatment until tumors reached peak size $\left(\mathrm{mm}^{2}\right) ; n=12$ mice/group. Mean time to peak tumor area compared by 1-way ANOVA with multiple comparisons; ${ }^{* *} P<0.001$. (D) Mean ( \pm SEM) peak tumor area $\left(\mathrm{mm}^{2}\right)$ in each mouse before onset of tumor control; $n=12$ mice/group. Mean tumor areas compared by 1-way ANOVA with multiple comparisons; ${ }^{*} P<0.5$. (E) Percentage survival of mice treated with early-, intermediate-, or late-culture Th17 cells; $n=12$ mice/group, representative of 3 independent experiments. Kaplan-Meier curve compared by log-rank test; ${ }^{* *} P<0.001$. (F) Onset of vitiligo in treated mice for each group; $n=12$ mice/group. Kaplan-Meier curve compared by log-rank test; ${ }^{*} P<0.01$. ns, not significant.

can indicate $\mathrm{T}$ cell fitness $(33,40)$. We observed that levels of CD28 were lower on early Th1 cells compared with early Th17 cells, and CD27 expression decreased on Th1 cells between day 7 and day 14, in contrast to Th17 cells (Figure 4E). Additionally, KLRG1 and the checkpoint inhibitor TIM3 were higher at each time point on Th1 cells versus Th17 cells. Interestingly, PD1 expression on early Th17 and Th1 cells was similar, but expression of this inhibitory molecule declined by day 14 on Th17 cells, while it increased on Th1 cells (Figure 4E).

To determine if expression of exhaustion/checkpoint molecules was merely an artifact of activation, we activated day-14 Th17 and Th1 cells using irradiated splenocytes bearing TRP-1 peptide. While CD69 expression increased substantially on Th17 cells, KLRG1 did not, and TIM3 and PD1 only increased marginally. However, expression of all these markers was higher on Th1 cells compared with Th17 cells with or without activation and was not affected by activation status (Supplemental Figure 4A).

An important difference between Th1 and Th17 cells is their tendency to undergo apoptosis and necrosis in culture. At day 7 ex vivo, $18 \%$ of Th1 cells were single positive for annexin $\mathrm{V}$ (indicating early entry into apoptosis), while $42 \%$ stained positive for propidium iodide (PI) (indicating either the end stages of apoptosis or necrosis). On the other hand, only $2 \%$ of Th17 cells were annexin $\mathrm{V}$ single positive or PI positive (Figure $4 \mathrm{~F}$ ). By day 14, 18\% of Th1 cells were annexin $\mathrm{V}$ single positive and $3 \%$ were PI positive, while Th17 cells had 4\% annexin V-single-positive and no PI-positive cells (Figure 4F). However, Th1 cells demonstrated much more sensitivity to activation-induced cell death than Th17 cells. Twelve hours after TCR engagement with an anti-CD3 antibody we observed that up to $55 \%$ of Th1 cells were annexin $\mathrm{V}$ single positive and $8 \%$ PI positive. Yet, Th17 cells remained resistant to apoptosis upon reactivation, demonstrated by only a slight increase in annexin $\mathrm{V}$ staining from $4 \%$ to $8 \%$ on these cells (Figure $4 \mathrm{~F}$ ). We observed a similar pattern of increased annexin $\mathrm{V}^{+}$cells in Th1 cells but not Th17 cells after TRP-1 and irradiated-splenocyte activation (Supplemental Figure 4B). These data suggest that Th17 cells maintain durable memory responses after long-term expansion due in part to their resistance to senescence and apoptosis, which may explain their improved ability to regress tumors compared with $\mathrm{Th} 1$ or $\mathrm{CD} 8^{+} \mathrm{T}$ cells.

Large numbers of Th17 cells cure mice with aggressive tumors. Up to this point, we tested the antitumor capacity of Th17 cells expanded for 1 to 3 weeks on a per-cell basis. This ignored the vast expansion of these cells by the end of 3 weeks of ex vivo expansion. We hypothesized that more Th17 cells $(>1$ $\times 10^{7}$ ) would be required to ablate large tumors and therefore would necessitate long-term expansion. 
A

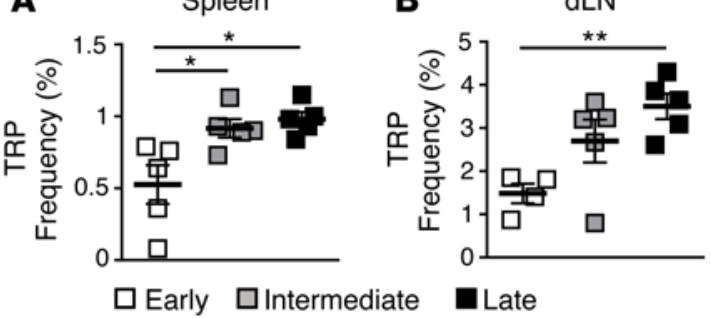

D
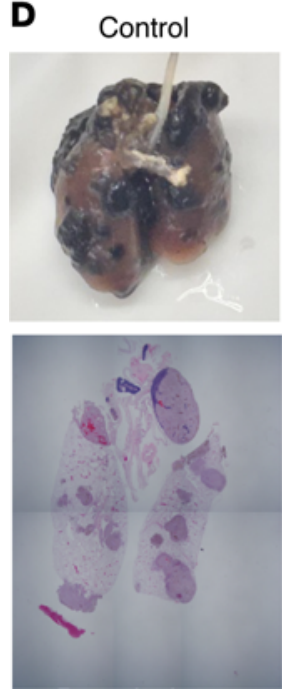

B

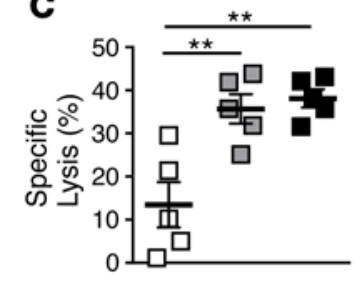

C

E

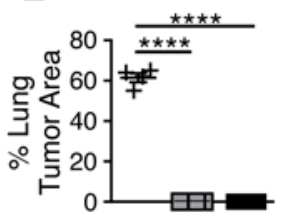

+ No Treatment

$\square$ Interm. $\square$ Late

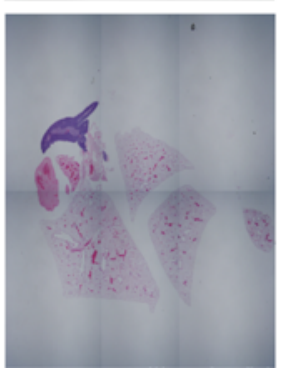

Late
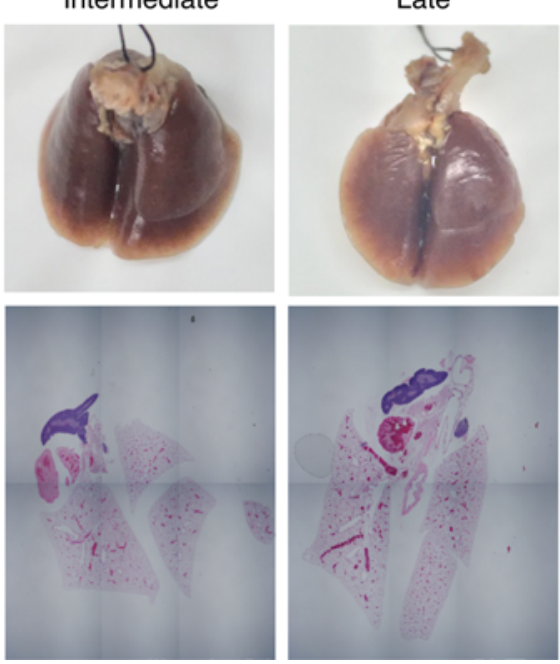

Figure 6. Infusing large numbers of Th17 cells after $\mathbf{2}$ or $\mathbf{3}$ weeks of expansion sets up longterm donor residence with enhanced memory response against antigen and tumor rechallenge. (A and B) Mean frequency ( \pm SEM) of donor Th17 cells in (A) spleens and (B) draining lymph nodes (dLNs) of surviving mice 40 days after treatment; $n=5$ mice/group. Mean frequencies compared by 1-way ANOVA with multiple comparisons; ${ }^{*} P<$ $0.05,{ }^{*} P<0.01$. TRP, CD4 ${ }^{+} T$ cells with a TCR specific for tyrosinase-related protein-1. (C) Memory recall assessed by in vivo lysis of peptide-pulsed splenocytes 40 days after treatment compared with control mice (mean percentage specific lysis \pm SEM); $n=5$ mice/group. Mean percentage specific lysis compared by 1-way ANOVA with multiple comparisons; ${ }^{* *} P<0.01$. (D) Macroscopic images of lungs and microscopic images of en-bloc sections from intermediate or late Th17-treated mice compared with control mice 21 days after rechallenge with $0.2 \times 10^{6}$ B16F10 cells injected i.v.; $n=2-6$ mice/group. (E) Quantification of percentage of lung taken up by tumor as determined by microscopic examination (mean $\pm \mathrm{SEM}$ ); $n=2-6$ mice/group. Mean percentage area compared by 1-way ANOVA with multiple comparisons; ${ }^{* * *} P<0.0001$.

We therefore capitalized on the high yield of Th17 cells, which still had remarkably preserved effector potency, to regress large, recalcitrant melanoma. To obtain differentially aged Th17 cell products and test their potential against large tumors, parallel cultures were grown from one Th17 polarization to early (day 7), intermediate (day 14), or late (day 18) time points, cryopreserved, and then split evenly among 12 mice/group. This protocol yielded mean treatment doses of $1.3 \times 10^{6}, 9.9 \times 10^{6}$, or $1.3 \times 10^{7}$ cells/mouse, respectively, to treat mice with large melanomas $\left(\sim 136 \mathrm{~mm}^{2}\right)$ (Figure $\left.5 \mathrm{~A}\right)$. As anticipated, we found that tumors regressed rapidly in mice treated with the high numbers of Th17 cells from either the intermediate or late cultures (Figure 5B). This was evidenced by the tumors in these mice reaching peak size earlier and having a smaller peak tumor size than mice treated with early Th17 cells (Figure 5, C and D). Conversely, mice treated with $1.3 \times 10^{6}$ early Th17 cells were unable to control tumor growth, with most mice succumbing to disease (Figure 5, B and E). The reduced antitumor efficacy of early Th17 cells was paralleled by a delay in onset of autoimmune vitiligo (Figure 5F). However, all mice given Th17 cells (at any amount or at any age) eventually developed a similar degree of vitiligo (Supplemental Figure 5A).

Infusing more Th17 cells from long-term expansion sets up long-lasting memory and protection against multiple rechallenges. As expected, higher frequencies of donor Th17 cells were detected in the spleen and tumor draining lymph nodes (dLNs) of mice infused with more Th17 cells (intermediate and late cultures) (Figure 6, A and B). Lower frequencies of early Th17 cells correlated with a decreased recall response to tumor antigen, as lysis of TRP-1 antigen-pulsed cells was significantly decreased in surviving mice treated with early Th17 cells compared with mice treated with intermediate- and late-culture Th17 cells (Figure 6C).

To determine if there was a difference between treating mice with intermediate or late Th17 cells, we administered a second dose of subcutaneous B16F10 to the cured animals. All animals from both treatment groups were protected from rechallenge (Supplemental Figure 5B), underscoring the therapeutic ability of Th17 cells to mediate durable tumor immunity. To further test the potency of this therapy, we rechallenged the mice again with a more aggressive metastatic model. B16F10 tumor cells were administered intravenously to mice to induce lung pseudometastases, which are far more challenging to treat than the subcutaneous tumors. Despite causing aggressive tumor outgrowth in the lungs of control mice, the lungs of all mice previously 
treated with either intermediate or late Th17 cells were protected from metastasis (Figure 6D and Supplemental Figure 5C). We performed histological analysis of the lungs to confirm our macroscopic observations of no metastatic tumor growth. Stepped sections were cut through whole-lung blocks (which included the heart and surrounding structures) from each group to determine the presence of any macroscopic or microscopic metastatic tumor deposits. In concordance with macroscopic findings, clear tumor nodules were noted in control lungs, while no melanoma deposits were seen in any of the other treatment groups (Figure $6 \mathrm{D}$ and Supplemental Figure 5C). In control mice, tumors occupied $\sim 61 \% \pm 4.5 \%$ of the total lung volume (Figure $6 \mathrm{E})$. These results highlight the capacity of long-term-expanded Th17 cells to eradicate tumors and provide durable protection from tumor metastasis.

Human Th17-polarized, but not Th1-polarized cells retain antitumor activity after expansion. As an important step towards translation of this work to clinical trials, we sought to determine if human Th17 cells possess the same undiminished capacity to clear tumors after long-term expansion as murine Th17 cells. We expected that human Th17 cells would remain highly therapeutic, while Th1 cells would decline in efficacy after expansion, similar to our observation with murine Th17 versus Th1 cells. To examine function, phenotype, and antitumor efficacy of human Th17 and Th1 cells, human CD4 ${ }^{+} \mathrm{T}$ cells from the peripheral blood of normal donors were polarized to either a Th17 or Th1 phenotype (see Methods). These 2 subsets were then expanded for 2 or more weeks after activation with anti-CD3 beads coated with ICOS and CD28, and redirected to express a chimeric antigen receptor (CAR) that recognizes mesothelin and signals through CD3 $\zeta$ and 4-1BB (MesoCAR). We also expanded bulk CD8 ${ }^{+} \mathrm{T}$ cells with CD3/CD28/ICOS beads and IL-2, and engineered them with the same MesoCAR construct. During expansion, Th17 cells became more polyfunctional with increased capacity to secrete IFN- $\gamma$ and IL-17A (Figure 7A). Interestingly, at all time points human Th17-polarized cells secreted more IFN- $\gamma$ than human Th1-polarized cells. This may be because among the cells derived from the peripheral blood were mature $\mathrm{T}$ cells that may have had prior functional profiles before polarization compared to the mainly naive T cells from TRP-1 transgenic mice.

However, Th17 polarization did induce high expression of the Th17-associated CCR6 chemokine, which was largely absent on Th1-polarized cells (Figure 7B). However, Th1 and Th17 cells both expressed similar levels of CXCR3, a chemokine receptor associated with Th1 cells. Thus, a large portion of Th17 cells became double positive for CCR6 and CXCR3 by the end of culture, a phenotypic marker of a highly functional nonclassical Th17/Th1 hybrid cell (Figure 7B) (41). Additionally, Th17 cells with the highest CCR6 expression also expressed CCR5 and CCR10, two chemokine receptors important for trafficking to inflamed tissues $(42,43)$ (Supplemental Figure 6, A and B). While present, these chemokine receptors were at lower concentrations on Th1 cells. Similar to murine $\mathrm{CD} 4^{+} \mathrm{T}$ cells, human Th1 cells expressed more CD62L at the beginning of culture than human Th17 cells, but by day 12, both subsets had less (though still ample) CD62L expression, indicating a progressive shift to an effector memory phenotype (Supplemental Figure 6C) - though not to the extent seen in murine Th17 cells (Figure 4A).

To test whether human Th17-polarized cells preserved antitumor activity during ex vivo expansion, we infused 7- or 12-day-expanded MesoCAR ${ }^{+}$Th17 cells $\left(2 \times 10^{6} /\right.$ mouse $)$ with 12-day-expanded MesoCAR ${ }^{+} \mathrm{CD}^{+} \mathrm{T}$ cells $\left(2 \times 10^{6} /\right.$ mouse $)$ into NSG mice with established M108 mesothelioma tumors. As a control, we also treated mice with 7- or 12-day-expanded MesoCAR ${ }^{+}$Th1 cells $\left(2 \times 10^{6} /\right.$ mouse $)$ with 12-day-expanded MesoCAR ${ }^{+} \mathrm{CD}^{+} \mathrm{T}$ cells $\left(2 \times 10^{6} /\right.$ mouse). We found that MesoCAR ${ }^{+} \mathrm{Th} 17$ cells eradicated human mesothelioma regardless of expansion time (Figure 7C). Conversely, treatment with 12-day-expanded MesoCAR ${ }^{+}$Th1 mediated poor antitumor activity compared with treatment with 7-dayexpanded MesoCAR ${ }^{+}$Th1 cells (Figure 7C). Importantly, Th17 cells could not mediate tumor immunity if engineered with a CAR against CD19 (Supplemental Figure 6D). This is not surprising, as mesothelioma tumors do not express CD19 antigen. Also, we tested the benefit of antigen-specific MesoCAR ${ }^{+} \mathrm{CD}^{+}$

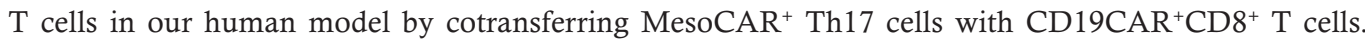
While antigen-specific Th17 cells with CD19CAR ${ }^{+} \mathrm{CD}^{+} \mathrm{T}$ cells controlled tumor growth well (and better than MesoCAR ${ }^{+} \mathrm{CD} 8^{+} \mathrm{T}$ cells transferred with $\mathrm{CD} 19 \mathrm{CAR}{ }^{+} \mathrm{Th} 17$ cells), we only observed curative responses when both the Th17 cells and $\mathrm{CD}^{+} \mathrm{T}$ cells were antigen specific against the correct target, mesothelin (Supplemental Figure 6D). Collectively, our data reveal that human Th17 cells retain their antitumor capacity when expanded long term ex vivo. Our human data confirm our findings that durable Th17 cells generated from ex vivo expansion (Figure 3), which dramatically eliminated large and metastatic melanoma tumors in mice (Figures 5 and 6), translates to human $\mathrm{T}$ cells with major implications for improving ACT clinical trials (Figure 7). 
A

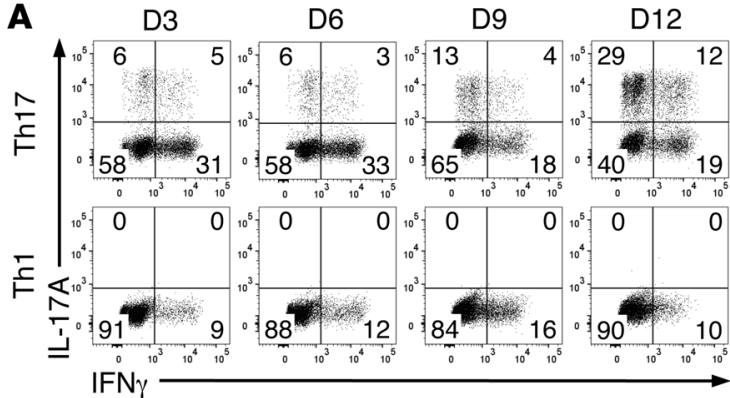

B

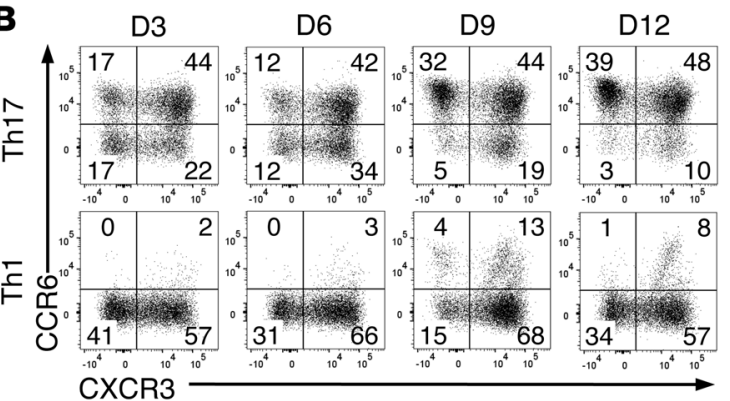

Figure 7. Human Th17 cells retain antitumor efficacy after 2 weeks of ex vivo expansion while Th1 cells do not. (A) IL-17A and IFN- $\gamma$ expression by human Th17- and Th1-polarized cells expanded for 3, 6, 9, or 12 days in vitro, representative of 2 normal donors. (B) CCR6 and CXCR3 expression on human Th17 and Th1 polarized cells expanded for $3,6,9$, or 12 days in vitro, representative of 2 normal donors. (C) M108 tumor burden of NSG mice treated with $2 \times 10^{6}$ seven- or twelve-day-expanded (D7 and D12, respectively) MesoCAR ${ }^{+} T$ 17 17 or MesoCAR ${ }^{+}$Th1 cells with $2 \times 10^{6}$ twelve-day-expanded MesoCAR ${ }^{+}$CD8 ${ }^{+}$ T cells, compared with no treatment; $n=8-11$ mice/group.
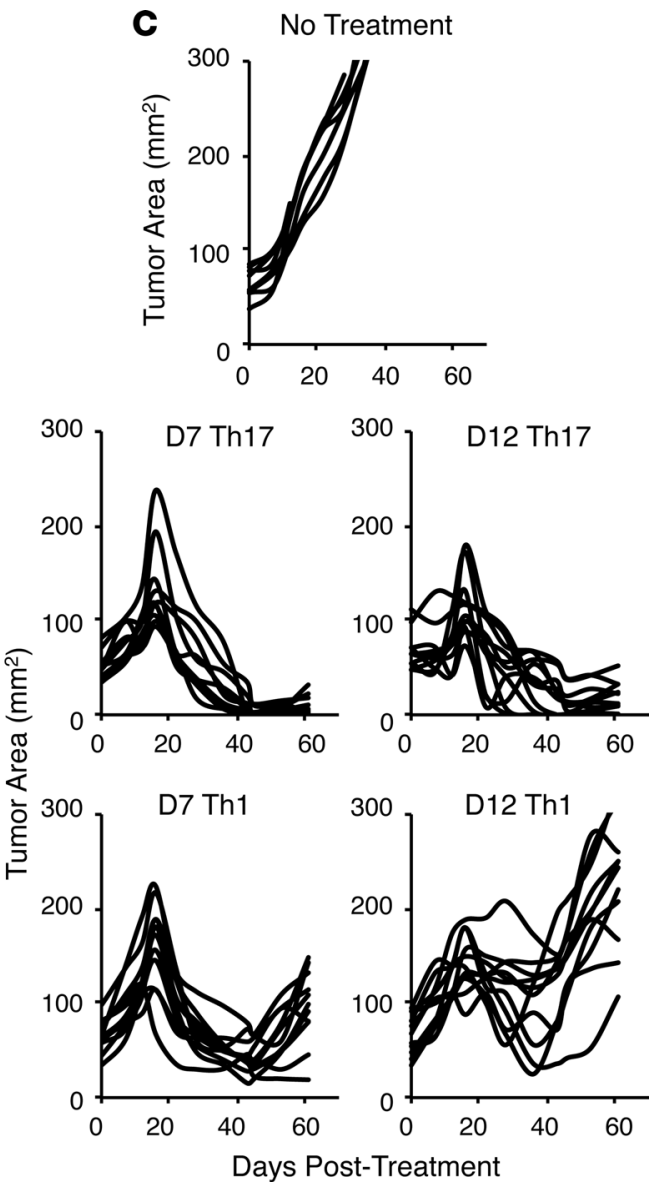

\section{Discussion}

ACT mediates objective response rates in $\sim 50 \%$ of cancer patients. This therapy relies on the infusion of large numbers of $\mathrm{T}$ cells $\left(10^{11}\right)$ to achieve this goal. Although $\mathrm{CD} 8^{+} \mathrm{T}$ cells are often used for ACT, they lose efficacy as they expand ex vivo. Herein, we discovered that Th17 cells have an undiminished capacity to regress tumors as they expand. Transferring $2 \times 10^{6} \mathrm{Th} 17$ cells expanded for 3 weeks ex vivo mediated antitumor immunity in mice similar to that mediated by infusing $2 \times 10^{6}$ younger Th17 cells expanded for 1 week. In contrast, neither Th1 nor $\mathrm{CD}^{+} \mathrm{T}$ cells retained antitumor efficacy after only 2 weeks of expansion. Thus, on a per-cell basis, the antitumor capacity of Th17 cells is more effective than $\mathrm{Th} 1$ or $\mathrm{CD}^{+} \mathrm{T}$ cells after long-term ex vivo expansion.

We hypothesized that the retention of antitumor efficacy by Th17 cells, coupled with their expansion to large numbers over 3 weeks, would allow us to treat large recalcitrant melanoma tumors $\left(\sim 175 \mathrm{~mm}^{2}\right)$ by treating mice using the entire cell product. Indeed, this high-dose Th17 therapy eradicated tumors in mice and mediated curative responses for 6 or more months. Also, our cured animals were protected from a subsequent tumor rechallenge both subcutaneously and intravenously to model aggressive lung metastases. In contrast, the few Th17 cells expanded for only 1 week were insufficient to clear even primary tumors. To our knowledge, this is the first report that concomitantly investigates 4 factors critical for ACT: (a) T cell age, (b) $\mathrm{T}$ cell yield, (c) $\mathrm{T}$ cell subset, and (d) level of tumor burden. Our findings are relevant, as they provide key insight into the potency of Th17 cells, which expand robustly without diminishing in quality.

We and other investigators have reported beneficial traits of Th17 cells for ACT $(18,27,28)$. Unlike Th1 cells, Th17 cells do not express KLRG1 or TIM3 during expansion, while cytokine receptors and costimulatory molecules are more abundant on Th17 cells than Th1 cells. Moreover, although resistance to apoptosis in Th17 cells has been reported $(27,44)$, we provide evidence that their resistance to apoptosis is continuous throughout in vitro culture. We suspect that these attributes also permit Th17 cells to persist in the harsh tumor microenvironment. 
Effector memory $\mathrm{CD}^{+} \mathrm{T}$ cells $\left(\mathrm{CD} 62 \mathrm{~L}^{-}\right)$are far less effective at regressing tumors when infused into mice or humans compared with their less differentiated counterparts, such as stem and central memory $\mathrm{CD} 8^{+} \mathrm{T}$ cells $(11,16,45)$. This observation begs the question: how can early or late effector memory Th17 cells that express nominal CD62L persist and still regress tumors in vivo? While previous studies indicate that Th17 cells have active $\beta$-catenin signaling after short-term in vitro expansion (28), our Western blot analysis of tumor-reactive Th17 cells found the transcription factor Tcf7 present in the nucleus at all time points during expansion. This observation is insightful, as Tcf7 is an essential protein in the Wnt/ $\beta$-catenin pathway that is critical for stem memory $\mathrm{T}$ cell self-renewal and was very recently found to be critical for formation of memory daughter cells through asymmetric T cell division $(28,29)$. Therefore, we posit that constitutive Tcf 7 signaling may partially explain why Th17 cells expand robustly in a Petri dish and persist long term in vivo. This finding might also explain why Th17 cells maintain their telomere length in vitro and in vivo, a property directly associated with $\mathrm{T}$ cell persistence in patients with enhanced survival in ACT trials $(1,13,14)$.

We found that Th17 cells are capable of directly lysing tumors. Both early- and late-expanded donor Th17 cells regressed melanoma independently of host $\mathrm{CD}^{+} \mathrm{T}$ cells, as antibody depletion of $\mathrm{CD} 8^{+} \mathrm{T}$ cells did not compromise treatment outcome. This is in alignment with a report by the Antony lab (46) that found tumor-reactive $\mathrm{CD} 4^{+} \mathrm{T}$ cells clear tumors in a class II-restricted manner in vivo. However, in our human CAR model, we found that infusion of both antigen-specific Th17 cells and CD8 ${ }^{+} \mathrm{T}$ cells was needed to mediate optimal antitumor responses, as MesoCAR ${ }^{+} \mathrm{Th} 17$ cells were unable to eradicate the tumors when coinfused with nonspecific $\mathrm{CD} 8^{+} \mathrm{T}$ cells (i.e., $\mathrm{CD} 19 \mathrm{CAR}{ }^{+} \mathrm{CD} 8^{+} \mathrm{T}$ cells) (Supplemental Figure 6D). This finding agrees with a study by the Dong laboratory (23), which found that depletion of endogenous $\mathrm{CD}^{+} \mathrm{T}$ cells diminished the antitumor response of Th17 cells, though it did not abrogate it completely. It is also important to appreciate that a possible confounding variable of depleting host $\mathrm{CD}^{+} \mathrm{T}$ cells in our TRP-1 ACT melanoma model would be the creation of space for the donor Th17 cells to engraft and better consume homeostatic cytokines $(38,47)$. Collectively, our data support a multilateral model where donor Th17 cells directly lyse tumors as well as cooperate with other immune cells, such as $\mathrm{CD} 8^{+} \mathrm{T}$ cells, to protect the host from tumor relapse long term.

Based on recent evidence supporting the benefit of shorter preparation time for ACT cell products $(11,48)$, cancer immunotherapists are devoting significant effort to reduce $\mathrm{T}$ cell expansion time ex vivo before infusion. A clinical trial at the NCI reported that they could generate young TILs that could mediate improved regression of metastatic melanoma. Yet, these TIL products were still expanded for a month or more ex vivo before being returned to the patient (49). Additionally, it is important to note that many parameters besides time contribute to $\mathrm{CD}^{+} \mathrm{T}$ cell exhaustion/loss of efficacy including: the number of times the T cells are restimulated; the growth factor cytokines used (IL-2 vs. IL-15 vs. IL-21); and the type of costimulatory signals (CD28, ICOS, or 4-1BB) delivered to expand the cells, all of which can alter their cellular bioenergetics $(19,50-54)$. In particular, IL-2 is the staple growth factor used to expand TIL and CAR-T cells for ACT. However, while this cytokine has potent effects on proliferation, IL-2 also differentiates $\mathrm{CD}^{+} \mathrm{T}$ cells to an effector or terminal status (55). These considerations give further credence to the use of T cells with stemness, such as Th17 cells, or pharmacologically altered CD8 ${ }^{+} \mathrm{T}$ cells $(16,17,28,56)$, both of which expand long term without reaching terminal differentiation.

Of clinical importance, human $\mathrm{CAR}^{+} \mathrm{Th} 17$-polarized cells also retain their antitumor potency after long-term expansion, while human $\mathrm{CAR}^{+} \mathrm{Th1}$-polarized cells do not. The antitumor activity of human $\mathrm{CAR}^{+} \mathrm{Th} 17$ cells in our mouse xenograft model of mesothelioma was superior to that of $\mathrm{CAR}^{+} \mathrm{Th} 1$ after either 7 or 12 days of ex vivo expansion. These findings recapitulate those in our TRP-1 melanoma model where murine Th17 cells mediate greater tumor immunity than TRP-1 Th1 cells. Of note, while the shortest reported TIL expansion protocols still require 1 month of ex vivo expansion, our data with 12-dayexpanded human Th17 CD4+ $\mathrm{T}$ cells is particularly relevant in the context of CAR therapies where many $\mathrm{T}$ cells can be quickly generated from peripheral blood within 1 or 2 weeks after apheresis.

In closing, Th17 cells offer promise for next-generation ACT trials, as they can: (a) expand rapidly in vitro for 3 weeks without requiring reactivation, (b) repopulate the host after infusion regardless of ex vivo expansion time, and (c) mediate potent destruction of large tumors when the total number of cells generated after 2 or more weeks of ex vivo culture is used. Th17 cell durability may also result in more consistent success in generating a therapeutic cell product, owing to a larger window of opportunity for securing potent $\mathrm{T}$ cells after ex vivo expansion. The resilience of Th17 cells in vitro may also simplify clinical preparation, rendering them more feasible to prepare for clinical trials in many cancer centers around the world, thereby extending ACT treatment to a wider patient population. 


\section{Methods}

\section{Mice and tumor lines}

C57BL/6J (B6), CD45.1 congenic B6, TRP-1 TCR transgenic mice, and NOD/scid/ $\gamma$ chain knockout (NSG) mice were purchased from Jackson Laboratories and housed and bred in the Medical University of South Carolina (MUSC) Hollings Cancer Center comparative medicine department. NSG mice were housed under specific pathogen-free conditions in microisolator cages and given autoclaved food and acidified water. Housing and experiments were conducted in accordance with MUSC's IACUC procedures. B16F10 (H-2b) melanoma tumor, a gift of the Nicholas Restifo lab at the NCI surgery branch, was maintained in culture media (RPMI 1640 with L-glutamine; 10\% FBS; $1 \%$ penicillin/streptomycin, nonessential amino acids, and Na pyruvate; and $0.1 \%$ 2-ME and Hepes). M108 xenograft tumors were a gift from the June lab at the University of Pennsylvania Philadelphia, Pennsylvania, USA. M108 were cultured and engrafted as described previously (57).

\section{T cell cultures}

TRP-1 cells. TRP-1 splenocytes were activated using 10-Gy-irradiated B6 splenocytes (feeder cells) pulsed with $1 \mu \mathrm{M}$ TRP-1 peptide and polarized to a Th17 phenotype at $3 \times 10^{6}$ cells $/ 2 \mathrm{ml}$ of cell media in 1 well of a 24-well plate with the following cocktail: $100 \mathrm{ng} / \mathrm{ml}$ recombinant human (rh) IL-6 (NIH repository), $100 \mathrm{ng} /$ $\mathrm{ml} \mathrm{rhIL}-21$ (Shenandoah), $30 \mathrm{ng} / \mathrm{ml}$ rhTGF- $\beta 1$ (Biolegend), $10 \mathrm{ng} / \mathrm{ml} \mathrm{rhIL}-1 \beta$ (NIH Repository), $10 \mu \mathrm{g} / \mathrm{ml}$ each of anti-mIFN- $\gamma$ (clone XMG1.2), anti-mouse IL-4 (anti-mIL-4) (clone 11B11), and anti-mIL-2 (clone JES6-1A12) (all BioXCell). Th1 polarization occurred under peptide activation with irradiated feeder cells with the following cocktail: $3 \mathrm{ng} / \mathrm{ml}$ recombinant mouse (rm) IL-12 (Shenandoah), $100 \mathrm{IU} / \mathrm{ml} \mathrm{rhIL}-2$ (NIH repository), and $10 \mu \mathrm{g} / \mathrm{ml}$ anti-mIL-4 (clone 11B11). Cultured cells were supplemented with new media containing $100 \mathrm{IU} / \mathrm{ml}$ rhIL-2 throughout expansion, then cryopreserved at time points indicated in 10\% DMSO in FBS. One day before transfer, cells were thawed and plated in cell media overnight and then transferred into mice.

Pmel-1 T cells. Pmel-1 splenocytes were activated using $1 \mu \mathrm{M}$ human gp100 peptide $+100 \mathrm{IU} \mathrm{rhIL}-2 / \mathrm{ml}$ and primed on day 1 with $40 \mathrm{ng} / \mathrm{ml}$ of rmIL-12. Cells were supplemented with culture media containing $100 \mathrm{IU}$ rhIL- $2 / \mathrm{ml}$ and expanded as indicated. $\mathrm{CD} 8^{+} \mathrm{T}$ cell cultures were in vitro activated with feeder cells and peptide 12 hours before transfer as described (39).

Human normal donor peripheral $T$ cells. To generate mesothelin-specific $\mathrm{T}$ cells, human $\mathrm{CD} 4^{+} \mathrm{T}$ cells were activated with CD3/CD28/ICOS-coated beads and programmed to a Th17 or Th1 phenotype and then transduced with a CAR that contained either an anti-mesothelin or anti-CD19 single-chain variable fragment $(\mathrm{scFv})$ fusion protein containing the TCR $\zeta$ signaling domain and 4-1BB that was generated as described previously (57) (gifts from the June lab). $\mathrm{CD}^{+} \mathrm{T}$ cells were polarized to the Th17 phenotype as follows: $10 \mathrm{ng} / \mathrm{ml} \mathrm{rhIL}-1 \beta, 10 \mathrm{ng} / \mathrm{ml} \mathrm{rhIL}-6,20 \mathrm{ng} / \mathrm{ml} \mathrm{rhIL}-23,10 \mu \mathrm{g} / \mathrm{ml}$ anti-hIL-4 (clone 11B11), and anti-hIFN- $\gamma$ (clone H22, eBioscience). Experiments were conducted with fetal calf serum containing endogenous sources of TGF- $\beta$. Th1 cells were polarized with $10 \mathrm{ng} / \mathrm{ml} \mathrm{rhIL}-12$ and $100 \mathrm{IU} / \mathrm{ml} \mathrm{rhIL}-2$. Cell cultures were maintained with $100 \mathrm{IU} / \mathrm{ml}$ of rhIL-2 and cells were expanded for up to 2 weeks. Likewise, human $\mathrm{CD}^{+} \mathrm{T}$ cells were activated with CD3/CD28/ICOS beads and redirected with either the antiMesoCAR or anti-CD19CAR constructs.

\section{$\mathrm{ACT}$}

B6 mice. B6 mice were given $4.5 \times 10^{5} \mathrm{~B} 16 \mathrm{~F} 10$ cells subcutaneously and tumors were allowed to establish between 5 and 12 days before ACT. One day before therapy, mice received nonmyeloablative 5 Gy total body irradiation. $\mathrm{T}$ cells were infused via tail vein. In the experiments with IL-12-primed CD8 ${ }^{+} \mathrm{T}$ cells, starting on the day of ACT, mice were given intraperitoneal injections of 600,000 IU rhIL-2 every other day for a total of 6 doses.

NSG mice. NSG mice were given $5 \times 10^{6} \mathrm{M} 108$ suspended in matrigel subcutaneously. Tumors were allowed to establish for 40 days prior to adoptive therapy. In all experiments, mice were randomized to treatment groups and tumor burden was monitored in blinded fashion using perpendicular caliper measurements and reported as tumor area $\left(\mathrm{mm}^{2}\right)$.

\section{Antibody depletion in vivo}

For cell depletion assays, mice were injected intraperitoneally with $100 \mu \mathrm{g}$ of either anti-CD8 depleting antibody (clone 53-6.72) or isotype control (clone 2A3) (both BioXCell) beginning the day before ACT and continuing weekly throughout the duration of the experiment. 


\section{Vitiligo autoimmunity score}

Severity of autoimmune vitiligo was scored after the phenotype stabilized. Depigmentation was rated based on a graduated scale from 1 to 5 with 1 being no loss of pigment (all black) and 5 being total loss of pigment (all white). Mice were scored in blinded fashion in triplicate.

\section{Tissue distribution assays}

Spleens from treated mice were harvested and mechanically disrupted using the tip of a syringe plunger. Cells were filtered through a wire mesh, red blood cells lysed with RBC lysis buffer (Biolegend), and then resuspended in cell media for analysis. Eyes and tumors were sectioned, and then incubated in $1 \mathrm{mg} / \mathrm{ml}$ collagenase type II (Life Technologies) at $37^{\circ} \mathrm{C}$ for 1 hour. Digested tissue was filtered, resuspended in cell media, and plated for assay. Before probing with antibodies, FC block (Biolegend) was applied to cells at 1 $\mu \mathrm{g} / 100 \mu \mathrm{l}$. Cell suspensions were then analyzed by flow cytometry.

\section{Flow cytometry, ELISA, and in situ flow}

Flow cytometry was performed with a BD FACSverse instrument. Antibodies used were: anti-mCD3\&eFluor450 (clone 17A2), anti-h/mROR $\gamma t-P E$ (clone AFKJS-9) (both eBioscience); anti-m4-1BB-Biotin (clone 17B5), anti-hCCR6-PECy7 (clone G034E3), anti-hCCR10-PE (clone 6588-5), anti-mCD25-FITC (clone 7D4), anti-mCD27-PECy7 (clone LG.3A10), anti-mCD28-PerCPCy5.5 (clone 37.51), anti-h/ mCD44-PerCPCy5.5 (clone IM7), anti-mCD69-PECy7 (clone HI.2F3), anti-mCD127-PE (clone A7R34), anti-hCXCR3-AF647 (clone G025H7), anti-mLAG3-PE (clone C9B7W), anti-mPD1-PerCPCy5.5 (clone 29F.1A12), Streptavidin-BV 421, anti-mTIM3-PE (clone B8.2C12) (all Biolegend); anti-hCCR5-V450 (clone 2D7/CCR5), anti-mCCR7-PE (clone 4B12), anti-hCD4-APCH7 (clone RPA-T4), anti-mCD4-APC/PE (clone RM4-5), anti-mCD45.1-APCCy7 (clone A20), anti-mCD45.2-V500 (clone 104), anti-mCD62L-APC (clone MEL-14), anti-mCD95-PECy7 (clone Jo2), anti-mICOS-Biotin (clone 7E.17G9), anti-mKLRG1APC (clone 2F1), anti-mNK1.1-PE (clone PK136), anti-h/mTbet-AF647 (clone 4B10), and anti-mVß14FITC (clone 14-2) (all BD). Apoptosis assays were performed with Biolegend's FITC Annexin V Apoptosis Detection Kit with PI, per the manufacturer's instructions. ELISAs for IL-17A, IL-17F, IL-22, IFN- $\gamma$, and IL-2 were performed using DuoSet ELISA kits (R\&D Systems) per the manufacturer's instructions. Telomere length was measured by in situ hybridization/flow cytometry using the Telomere PNA Kit/FITC via Flow Cytometry (Dako) per the manufacturer's instructions.

\section{In vivo cytotoxicity assay}

C57BL/6 splenocytes were incubated with either TRP peptide or OVA peptide residues 323-339 (OTII) (Invivogen) and then labeled with a low level $(0.5 \mu \mathrm{M})$ or high level $(5 \mu \mathrm{M})$ of cell trace violet (CTV) (Thermo Fisher Scientific), respectively. TRP- and OTII-pulsed splenocytes were mixed at an equal ratio and then injected intravenously into mice previously treated with Th17 cells or untreated control mice. After 12 hours, spleens were harvested from mice and the ability of donor cells to lyse TRP-pulsed splenocytes versus OTII-pulsed splenocytes was assessed via flow cytometry and reported as percentage specific lysis using the following equation: $\%$ specific lysis $=(1-$ ratio of no-T-cellcontrol mice] $/$ [ratio of ACT mice] $) \times 100$, where the ratio $=\%$ OTII $/ \%$ TRP).

\section{Western blot}

Nuclear protein from Th17 cells cultured for 7, 14, or 21 days was extracted via the NE-PER Nuclear and Cytoplasmic Extraction kit (Thermo Fisher Scientific) and then analyzed by Western blot. The following primary antibodies were used: rabbit anti-h/mTCF7 (Abcam, clone EPR2035); rabbit anti-h/mHistone H3 (clone D1H2), rabbit anti-h/mSTAT1 polyclonal (catalog 9172), rabbit anti-h/mSTAT3 (clone D3Z2G) (all Cell Signaling Technology); rat anti-h/mROR $\gamma \mathrm{t}$ (clone AFKJS-9), and mouse anti-h/mTbet (clone 4B10) (both eBioscience).

\section{Histology}

For lung histology and morphometry, lungs were inflated en bloc and fixed with $4 \%$ buffered paraformaldehyde at a constant hydrostatic pressure of $25 \mathrm{~cm}$ and further immersed in fixative for 48 hours. The inflated lungs were embedded in paraffin and 4- $\mathrm{m}$ sagittal stepped sections were stained with H\&E for histological evaluation. In total, 10 stepped sections from each lung were analyzed to calculate the volume of metastatic 
tumor in each group using an automated motorized stage Olympus BX61 microscope to generate lung superimages using Visiopharm software. The total area of lung and tumor metastases was calculated using Visiopharm image acquisition software by an observer blinded to the experimental groups.

\section{Statistics}

Kaplan-Meier survival curves were assessed for significance using a log-rank test between treatment groups. Comparisons between 2 groups were analyzed using a 2-tailed Student's $t$ test with Welch's correction for parametric distribution or Mann-Whitney signed-rank test for nonparametric distribution. For comparisons between multiple groups, a 1-way ANOVA was performed followed by multiple comparisons. $P$ values less than 0.05 were considered significant.

\section{Study approval}

Studies were approved by the IACUC of the MUSC Animal Resource Center (number 3039). De-identified human peripheral blood mononuclear cells were collected under approval of the MUSC IRB (pro13570). Human T cells were engineered via approval from the MUSC institutional biosafety committee (number 2335).

\section{Author contributions}

$\mathrm{JB}$ and $\mathrm{CP}$ were responsible for conception and design of this project. Methodology was developed by JB, BR, CA, AK, LG, and CP. Data were acquired by JB, AK, CA, and CP. Interpretation and analysis of data were peformed by JB, M. Nelson, KM, SB, CA, and CP. The following participated in writing, review, and revision of the manuscript: $\mathrm{JB}, \mathrm{MN}, \mathrm{KM}, \mathrm{SB}, \mathrm{BR}, \mathrm{LG}, \mathrm{CP}$. CP was responsible for administrative, technical, and material support as well as supervision of this study.

\section{Acknowledgments}

We thank Logan Huff and Hannah Knochelmann for experimental support and Kent Armeson for help in statistical analysis of this work. This research was supported in part by the Hollings Cell Evaluation \& Therapy, and Hollings Biostatistics Shared Resources, Hollings Cancer Center, MUSC (P30 CA138313).

This work was supported by NIH fellowship grant F30 CA200272 and NIH training grant T32 GM008716 to J.S. Bowers, a Jeane B. Kempner Foundation grant and ACS Postdoctoral fellowship (122704-PF-13-084-01-LIB) grant to M.H. Nelson, an NIH fellowship grant F31 CA192787 to S.R. Bailey, and NCI grants R01 CA175061 and R01 CA208514, KL2 South Carolina Clinical \& Translational Research grant UL1 TR000062, ACS-IRG grant 016623-004, and MUSC start-up funds to C.M. Paulos.

Address correspondence to: Jacob S. Bowers or Chrystal M. Paulos, Hollings Cancer Center, Medical University of South Carolina, 86 Jonathan Lucas Street, Room 606B MSC509, South Carolina 29425, USA. Phone: 843.792.8926; E-mail: bowersjs@musc.edu (J.S. Bowers). Phone: 843.792.3210; E-mail: paulos@musc.edu (C.M. Paulos).

ADMK's present address is: Miltenyi Biotec, Bergisch Gladbach, Germany.

1. Robbins PF, et al. Cutting edge: persistence of transferred lymphocyte clonotypes correlates with cancer regression in patients receiving cell transfer therapy. J Immunol. 2004;173(12):7125-7130.

2. Rosenberg SA. Cell transfer immunotherapy for metastatic solid cancer--what clinicians need to know. Nat Rev Clin Oncol. 2011;8(10):577-585

3. Phan GQ, Rosenberg SA. Adoptive cell transfer for patients with metastatic melanoma: the potential and promise of cancer immunotherapy. Cancer Control. 2013;20(4):289-297.

4. Rosenberg SA, Spiess P, Lafreniere R. A new approach to the adoptive immunotherapy of cancer with tumor-infiltrating lymphocytes. Science. 1986;233(4770):1318-1321.

5. Rosenberg SA, et al. Use of tumor-infiltrating lymphocytes and interleukin-2 in the immunotherapy of patients with metastatic melanoma. A preliminary report. NEngl J Med. 1988;319(25):1676-1680.

6. Rosenberg SA, et al. Treatment of patients with metastatic melanoma with autologous tumor-infiltrating lymphocytes and interleukin 2. J Natl Cancer Inst. 1994;86(15):1159-1166.

7. Jotereau F, et al. High-fold expansion of human cytotoxic T-lymphocytes specific for autologous melanoma cells for use in immunotherapy. J Immunother. 1991;10(6):405-411.

8. Yee $\mathrm{C}$, et al. Adoptive $\mathrm{T}$ cell therapy using antigen-specific $\mathrm{CD} 8^{+} \mathrm{T}$ cell clones for the treatment of patients with metastatic mela- 
noma: in vivo persistence, migration, and antitumor effect of transferred T cells. Proc Natl Acad Sci USA. 2002;99(25):16168-16173.

9. Chapuis AG, et al. Transferred melanoma-specific $C D 8^{+} \mathrm{T}$ cells persist, mediate tumor regression, and acquire central memory phenotype. Proc Natl Acad Sci USA. 2012;109(12):4592-4597.

10. Mackensen A, Meidenbauer N, Vogl S, Laumer M, Berger J, Andreesen R. Phase I study of adoptive T-cell therapy using antigen-specific CD8 ${ }^{+} \mathrm{T}$ cells for the treatment of patients with metastatic melanoma. J Clin Oncol. 2006;24(31):5060-5069.

11. Gattinoni L, et al. Acquisition of full effector function in vitro paradoxically impairs the in vivo antitumor efficacy of adoptively transferred CD8 ${ }^{+}$T cells. J Clin Invest. 2005;115(6):1616-1626.

12. Díaz-Montero CM, et al. Synergy of brief activation of CD8 T-cells in the presence of IL-12 and adoptive transfer into lymphopenic hosts promotes tumor clearance and anti-tumor memory. Am J Cancer Res. 2011;1(7):882-896.

13. Zhou J, Shen X, Huang J, Hodes RJ, Rosenberg SA, Robbins PF. Telomere length of transferred lymphocytes correlates with in vivo persistence and tumor regression in melanoma patients receiving cell transfer therapy. J Immunol. 2005;175(10):7046-7052.

14. Shen X, et al. Persistence of tumor infiltrating lymphocytes in adoptive immunotherapy correlates with telomere length. $J$ Immunother. 2007;30(1):123-129.

15. Crompton JG, Sukumar M, Restifo NP. Uncoupling T-cell expansion from effector differentiation in cell-based immunotherapy. Immunol Rev. 2014;257(1):264-276.

16. Gattinoni L, et al. A human memory T cell subset with stem cell-like properties. Nat Med. 2011;17(10):1290-1297.

17. Crompton JG, et al. Akt inhibition enhances expansion of potent tumor-specific lymphocytes with memory cell characteristics. Cancer Res. 2015;75(2):296-305.

18. Muranski P, et al. Tumor-specific Th17-polarized cells eradicate large established melanoma. Blood. 2008;112(2):362-373.

19. Paulos CM, et al. The inducible costimulator (ICOS) is critical for the development of human T(H)17 cells. Sci Transl Med 2010;2(55):55ra78.

20. Bailey SR, Nelson MH, Himes RA, Li Z, Mehrotra S, Paulos CM. Th17 cells in cancer: the ultimate identity crisis. Front Immunol. 2014;5:276

21. Shedlock DJ, Shen H. Requirement for CD4 T cell help in generating functional CD8 T cell memory. Science. 2003;300(5617):337-339.

22. Ekkens MJ, et al. Th1 and Th2 cells help CD8 T-cell responses. Infect Immun. 2007;75(5):2291-2296.

23. Martin-Orozco N, et al. T helper 17 cells promote cytotoxic T cell activation in tumor immunity. Immunity. 2009;31(5):787-798

24. Tran E, et al. Cancer immunotherapy based on mutation-specific CD4 ${ }^{+} \mathrm{T}$ cells in a patient with epithelial cancer. Science. 2014;344(6184):641-645.

25. Cohen PA, Peng L, Plautz GE, Kim JA, Weng DE, Shu S. CD4+ T cells in adoptive immunotherapy and the indirect mechanism of tumor rejection. Crit Rev Immunol. 2000;20(1):17-56.

26. Perez-Diez A, et al. CD4 cells can be more efficient at tumor rejection than CD8 cells. Blood. 2007;109(12):5346-5354

27. Kryczek I, et al. Human TH17 cells are long-lived effector memory cells. Sci Transl Med. 2011;3(104):104ra100.

28. Muranski P, et al. Th17 cells are long lived and retain a stem cell-like molecular signature. Immunity. 2011;35(6):972-985.

29. Nish SA, et al. CD4 ${ }^{+} \mathrm{T}$ cell effector commitment coupled to self-renewal by asymmetric cell divisions. J Exp Med. 2017;214(1):39-47.

30. Nelson $\mathrm{MH}$, et al. The inducible costimulator augments Tc17 cell responses to self and tumor tissue. J Immunol. 2015;194(4):1737-1747.

31. Johnson CB, et al. Effector CD8 ${ }^{+}$T-cell engraftment and antitumor immunity in lymphodepleted hosts is IL7R $\alpha$ dependent. Cancer Immunol Res. 2015;3(12):1364-1374.

32. Hendriks J, Gravestein LA, Tesselaar K, van Lier RA, Schumacher TN, Borst J. CD27 is required for generation and long-term maintenance of T cell immunity. Nat Immunol. 2000;1(5):433-440.

33. Appay V, van Lier RA, Sallusto F, Roederer M. Phenotype and function of human T lymphocyte subsets: consensus and issues. Cytometry A. 2008;73(11):975-983

34. Palmer DC, et al. Effective tumor treatment targeting a melanoma/melanocyte-associated antigen triggers severe ocular autoimmunity. Proc Natl Acad Sci USA. 2008;105(23):8061-8066.

35. Basu R, Hatton RD, Weaver CT. The Th17 family: flexibility follows function. Immunol Rev. 2013;252(1):89-103.

36. Mukasa R, et al. Epigenetic instability of cytokine and transcription factor gene loci underlies plasticity of the $\mathrm{T}$ helper 17 cell lineage. Immunity. 2010;32(5):616-627.

37. Bowers JS, et al. Dendritic cells in irradiated mice trigger the functional plasticity and antitumor activity of adoptively transferred Tc17 cells via IL12 signaling. Clin Cancer Res. 2015;21(11):2546-2557.

38. Gattinoni L, et al. Removal of homeostatic cytokine sinks by lymphodepletion enhances the efficacy of adoptively transferred tumor-specific CD8 ${ }^{+}$T cells. J Exp Med. 2005;202(7):907-912.

39. Klebanoff CA, Yu Z, Hwang LN, Palmer DC, Gattinoni L, Restifo NP. Programming tumor-reactive effector memory CD8 ${ }^{+} \mathrm{T}$ cells in vitro obviates the requirement for in vivo vaccination. Blood. 2009;114(9):1776-1783.

40. Voehringer D, Koschella M, Pircher H. Lack of proliferative capacity of human effector and memory T cells expressing killer cell lectinlike receptor G1 (KLRG1). Blood. 2002;100(10):3698-3702.

41. Acosta-Rodriguez EV, et al. Surface phenotype and antigenic specificity of human interleukin 17-producing $\mathrm{T}$ helper memory cells. Nat Immunol. 2007;8(6):639-646.

42. Samson M, Labbe O, Mollereau C, Vassart G, Parmentier M. Molecular cloning and functional expression of a new human CC-chemokine receptor gene. Biochemistry. 1996;35(11):3362-3367.

43. Homey B, et al. CCL27-CCR10 interactions regulate T cell-mediated skin inflammation. Nat Med. 2002;8(2):157-165

44. Yu Y, et al. Abundant c-Fas-associated death domain-like interleukin-1-converting enzyme inhibitory protein expression determines resistance of T helper 17 cells to activation-induced cell death. Blood. 2009;114(5):1026-1028.

45. Berger C, Jensen MC, Lansdorp PM, Gough M, Elliott C, Riddell SR. Adoptive transfer of effector CD8 ${ }^{+}$T cells derived from central memory cells establishes persistent T cell memory in primates. J Clin Invest. 2008;118(1):294-305.

46. Xie Y, et al. Naive tumor-specific CD4(+) T cells differentiated in vivo eradicate established melanoma. J Exp Med. 2010;207(3):651-667. 
47. Klebanoff CA, Khong HT, Antony PA, Palmer DC, Restifo NP. Sinks, suppressors and antigen presenters: how lymphodepletion enhances T cell-mediated tumor immunotherapy. Trends Immunol. 2005;26(2):111-117.

48. Klebanoff CA, et al. Memory T cell-driven differentiation of naive cells impairs adoptive immunotherapy. J Clin Invest. 2016;126(1):318-334.

49. Tran KQ, et al. Minimally cultured tumor-infiltrating lymphocytes display optimal characteristics for adoptive cell therapy J Immunother. 2008;31(8):742-751.

50. Hinrichs CS, et al. IL-2 and IL-21 confer opposing differentiation programs to CD8 ${ }^{+} \mathrm{T}$ cells for adoptive immunotherapy. Blood 2008;111(11):5326-5333.

51. Hinrichs CS, et al. Human effector $\mathrm{CD} 8^{+} \mathrm{T}$ cells derived from naive rather than memory subsets possess superior traits for adoptive immunotherapy. Blood. 2011;117(3):808-814.

52. Klebanoff CA, et al. IL-15 enhances the in vivo antitumor activity of tumor-reactive CD8 ${ }^{+}$T cells. Proc Natl Acad Sci USA 2004;101(7):1969-1974.

53. Kawalekar OU, et al. Distinct signaling of coreceptors regulates specific metabolism pathways and impacts memory development in CAR T cells. Immunity. 2016;44(2):380-390.

54. Buck MD, et al. Mitochondrial dynamics controls T cell fate through metabolic programming. Cell. 2016;166(1):63-76.

55. Verdeil G, Puthier D, Nguyen C, Schmitt-Verhulst AM, Auphan-Anezin N. STAT5-mediated signals sustain a TCR-initiated gene expression program toward differentiation of CD8 T cell effectors. J Immunol. 2006;176(8):4834-4842.

56. van der Waart AB, et al. Inhibition of Akt signaling promotes the generation of superior tumor-reactive T cells for adoptive immunotherapy. Blood. 2014;124(23):3490-3500.

57. Carpenito $\mathrm{C}$, et al. Control of large, established tumor xenografts with genetically retargeted human T cells containing CD28 and CD137 domains. Proc Natl Acad Sci USA. 2009;106(9):3360-3365. 